\title{
Localized Endomorphisms of the Chiral Ising Model
}

\author{
Jens Böckenhauer \\ II. Institut für Theoretische Physik, Universität Hamburg \\ Luruper Chaussee 149, D-22761 Hamburg
}

August 23, 2018

\begin{abstract}
Based on the treatment of the chiral Ising model by Mack and Schomerus, we present examples of localized endomorphisms $\varrho_{1}^{\text {loc }}$ and $\varrho_{1 / 2}^{\text {loc }}$. It is shown that they lead to the same superselection sectors as the global ones in the sense that unitary equivalence $\pi_{0} \circ \varrho_{1}^{\text {loc }} \cong \pi_{1}$ and $\pi_{0} \circ \varrho_{1 / 2}^{\text {loc }} \cong \pi_{1 / 2}$ holds. Araki's formalism of the selfdual CAR algebra is used for the proof. We prove local normality and extend representations and localized endomorphisms to a global algebra of observables which is generated by local von Neumann algebras on the punctured circle. In this framework, we manifestly prove fusion rules and derive statistics operators.
\end{abstract}

\section{Introduction}

In local quantum field theory one considers a Hilbert space $\mathcal{H}$ of physical states which decomposes into orthogonal subspaces $\mathcal{H}_{J}$ (superselection sectors) so that observables do not make transitions between the sectors. The subspaces $\mathcal{H}_{J}$ carry inequivalent, irreducible representations of the observable algebra $\mathcal{A}$, possibly with some multiplicities $[18]$. Among the superselection sectors, there is a distinguished sector $\mathcal{H}_{0}$ which contains the vacuum vector $\left|\Omega_{0}\right\rangle$ and carries the vacuum representation $\pi_{0}$.

The starting point in the algebraic approach to quantum field theory is the observable algebra $\mathcal{A}$ which is usually defined as the $C^{*}$-inductive limit of the net of local von Neumann algebras $\{\mathcal{A}(\mathcal{O}), \mathcal{O} \in \mathcal{K}\}$, where $\mathcal{K}$ denotes the set of open double cones in $D$ dimensional Minkowski space. The net is assumed to satisfy the Haag-Kastler-axioms. In general, the observable algebra $\mathcal{A}$ admits a lot of inequivalent representations. Therefore one has to find an appropriate selection criterion which rules out the physically non-relevant representations. Doplicher, Haag and Roberts [10, 11, 17] developed the theory of locally generated sectors; they suggested that one has to consider only those representations $\pi_{J}$ which become equivalent to the vacuum representation in the restriction to the causal complement $\mathcal{O}^{\prime}$ of any sufficiently large double cone $\mathcal{O} \in \mathcal{K}$. That means that for a representation $\pi_{J}$ satisfying the DHR criterion, there exists for each sufficiently large double cone $\mathcal{O}$ a unitary $V: \mathcal{H}_{0} \rightarrow \mathcal{H}_{J}$ such that

$$
\pi_{J}(A)=V \pi_{0}(A) V^{*}, \quad A \in \mathcal{A}\left(\mathcal{O}^{\prime}\right) .
$$


The DHR criterion leads to the characterization of superselection sectors by localized endomorphisms: Usually $\mathcal{A}$ and $\pi_{0}(\mathcal{A})$ are identified, and one defines

$$
\varrho_{J}(A)=V^{*} \pi_{J}(A) V, \quad A \in \mathcal{A} .
$$

Then $\varrho_{J}$ is an endomorphism of the observable algebra and it is localized in $\mathcal{O}$ in the sense that $\varrho_{J}(A)=A$ for all $A \in \mathcal{A}\left(\mathcal{O}^{\prime}\right)$. Moreover, $\pi_{0} \circ \varrho_{J}$ is a representation of $\mathcal{A}$ in $\mathcal{H}_{0}$ which is equivalent to $\pi_{J}$. The use of DHR endomorphisms allows to extract all physical information out of the vacuum sector and to work without charged, unobservable fields. It is another advantage that endomorphisms can be composed; it is possible to formulate fusion rules in terms of equivalence classes of localized endomorphisms.

Unfortunately, it seems to be very difficult to construct these endomorphisms explicitly in models. Although the conformal field theory has turned out to be an interesting and fruitful area of application of the DHR program, examples of localized endomorphisms which generate charged sectors are known explicitly only for a rather small number of models, e.g. the $U(1)$ current algebra on the circle [8]. Endomorphisms have been constructed for Level 1 WZW models [16] and, before that, for the chiral Ising model 223, 24], however, they are in no sense localized. Mack and Schomerus had already described the construction of localized endomorphisms for the chiral Ising model in [23], but it has not yet been proven that they lead to the same sectors as the global ones and, in particular, that they lead to irreducible representations. This is done in the present paper.

In two-dimensional conformal field theory one considers as basic observable the stress energy tensor which generates the space time symmetry. Its light cone components $T_{ \pm}\left(z_{ \pm}\right)$live separately on the compactified light cone variables $z_{ \pm} \in S^{1}$, one deals with chiral fields. Treating each component for its own, the stress energy tensor has well-known commutation relations, fixed up to a constant $c$ 22, 22; the stress energy tensor generates the Virasoro algebra Vir. In the case $c=\frac{1}{2}$ (Ising model) the Virasoro algebra admits three inequivalent positive energy representations $\pi_{J}, J=0, \frac{1}{2}$, 1, which are lowest weight representations; $\pi_{0}$ is identified to be the vacuum representation. In the chiral Ising model, the stress energy tensor can be built of a free fermion field, the Majorana field [23, 22]. Smearing out the Majorana field with test functions having support in a proper subinterval $I \subset S^{1}$ and considering bilinear expressions of it, these objects generate the local $C^{*}$-algebra $\mathcal{A}(I)$ of observables. Such local algebras $\mathcal{A}(I)$ generate a global observable algebra $\mathcal{A}_{\text {univ }}^{C^{*}}$. Unfortunately, the Virasoro generators are not in $\mathcal{A}_{\text {univ }}^{C^{*}}$, but they are formal (unbounded) limits of elements in $\mathcal{A}_{\text {univ }}^{C^{*}}$. Mack and Schomerus [23, 24] presented endomorphisms $\varrho_{J}$ such that $\pi_{0} \circ \varrho_{J} \cong \pi_{J}, J=\frac{1}{2}, 1$ is fulfilled. But, as already mentioned, these endomorphisms are not localized, i.e. there is no interval $I^{\prime} \neq \emptyset$ such that their action is trivial on $\mathcal{A}\left(I^{\prime}\right)$. In this paper we present examples $\varrho_{1 / 2}^{\text {loc }}$, $\varrho_{1}^{\text {loc }}$ of localized endomorphisms which are unitarily equivalent to those global ones in composition with the vacuum representation. However, our construction slightly differs from the formalism used by Mack and Schomerus.

Since the set $\mathcal{J}$ of proper subintervals on the circle is not directed, a global algebra cannot be defined as the $C^{*}$-inductive limit of the system $\{\mathcal{A}(I), I \in \mathcal{J}\}$. The global algebra $\mathcal{A}_{\text {univ }}^{C^{*}}$ has to be considered as the algebra generated freely by all local algebras $\mathcal{A}(I)$. This is the universal algebra (but generated by local $C^{*}$ - 
algebras instead of von Neumann algebras) in the sense of Fredenhagen, Rehren and Schroer 14, 15. Its center is nontrivial, generated by a unitary element $Y$. The $C^{*}$-algebra of the punctured circle $\mathcal{A}\left(I_{\zeta}\right)$ where $I_{\zeta}=S^{1} \backslash\{\zeta\}, \zeta \in S^{1}$ an arbitrary point, has a trivial center and $\mathcal{A}_{\text {univ }}^{C^{*}}$ is generated by $\mathcal{A}\left(I_{\zeta}\right)$ and $Y$. The local algebras $\mathcal{A}(I)$ are even subalgebras of (selfdual) CAR algebras over spaces $L^{2}(I)$. Also the global observable algebra $\mathcal{A}_{\text {univ }}^{C^{*}}$ is the even subalgebra of a global field algebra, the universal Majorana algebra Maj. It has the structure of the direct sum of two selfdual CAR algebras over $L^{2}\left(S^{1}\right)$. Alternatively, it may be regarded as the algebra which is generated by an anticommuting universal Majorana field living on the double cover $\tilde{S}^{1}$ of the unit circle [23, 24]. For recovering the local algebras $\mathcal{A}(I)$ as even subalgebras of Maj by explicit construction, we have to fix an arbitrary reference point $\zeta$ ("point at infinity") on the circle.

The non-trivial center of the global algebra $\mathcal{A}_{\text {univ }}^{C^{*}}$ implies that its irreducible representations can no longer be faithful. This leads to some deviations from the customary DHR program. In particular, the vacuum representation $\pi_{0}$ of $\mathcal{A}_{\text {univ }}^{C^{*}}$ cannot be faithful. There is another difference between the formalism used by Mack and Schomerus and the common DHR framework: The local algebras $\mathcal{A}(I)$ are defined as $C^{*}$-algebras instead of von Neumann algebras. But the use of von Neumann algebras is crucial for the analysis of statistics and fusion. On the other hand, if one works with local von Neumann algebras (weak closures of $\mathcal{A}(I)$ in the vacuum representation), the universal algebra becomes even larger, in particular, its center is larger than that of $\mathcal{A}_{\text {univ }}^{C^{*}}$, generated by $Y$. Such a universal algebra appears to be hard to handle.

During our investigations, it turned out to be much more comfortable to formulate the theory on the punctured circle. Such a formulation is possible because in our theory Haag duality remains valid on the punctured circle. Having fixed a "point at infinity" $\zeta \in S^{1}$, the set $\mathcal{J}_{\zeta}$ of those open intervals such that $\zeta$ is not contained in their closures ("finite intervals") is directed by inclusion. So the theory can be developed close to the DHR program. We define local von Neumann algebras $\mathcal{R}(I)=\pi_{0}(\mathcal{A}(I))^{\prime \prime}$, and the net $\left\{\mathcal{R}(I), I \in \mathcal{J}_{\zeta}\right\}$ generates a global $C^{*}$-algebra $\mathfrak{A}_{\zeta}$ in the natural way; $\mathfrak{A}_{\zeta}$ may be regarded as the algebra of quasilocal observables. The representations $\pi_{J}$ and the localized endomorphisms $\varrho_{J}^{\text {loc }}$ we present are at first defined on local $C^{*}$-algebras $\mathcal{A}(I)$. We show that they possess an extension to the net of von Neumann algebras. A local normality relation is used for the proof. Using some results of CAR theory, we establish that indeed unitary equivalence $\pi_{0} \circ \varrho_{J}^{\text {loc }} \cong \pi_{J}$ holds. Finally, we manifestly prove the Ising fusion rules in terms of equivalence classes of localized endomorphisms, and we construct statistics operators and a left inverse.

With respect to the proof of fusion rules, we believe to close a gap left in [23, 24]. Mack and Schomerus had already proven that their global endomorphisms obey Ising fusion rules. But caused by the use of local $C^{*}$-algebras and a non-faithful vacuum representation, this result could not be generalized to all endomorphisms, which lead to equivalent representations. (There are counterexamples.) The existence of unitary intertwiners in the observable algebra, being essential for such a generalization, is not guaranteed if one does not work with von Neumann algebras. However, establishing a theory based on local von Neumann algebras on the punctured circle, we close the gap. But it should be mentioned that there exist also successful methods for proving conformal field theory fusion rules without the use of localized endomorphisms, e.g. 21, 31]. 
Our paper is organized as follows. We present Majorana fields, local $C^{*}$ algebras of observables and the global algebra $\mathcal{A}_{\text {univ }}^{C^{*}}$ in Section 2. Using some ideas of Szlachányi [30], we discuss the origin of its central element $Y$. We introduce the universal Majorana algebra Maj, we describe the representation theory of Maj and $\mathcal{A}_{\text {univ }}^{C^{*}}$ and we introduce the Mack-Schomerus endomorphisms. Section 3 begins with a brief recapitulation of the CAR theory and some results we need. Next we describe the representation theory of Maj and $\mathcal{A}_{\text {univ }}^{C^{*}}$ in view of Araki's selfdual CAR algebra and quasifree states. We discuss the restriction to the algebra $\mathcal{A}\left(I_{\zeta}\right)$ of the punctured circle. Then we introduce our examples of localized endomorphisms and we analyze the induced representations. In Section 4 we discuss the extension of representations and localized endomorphisms to local von Neumann algebras and to the global algebra $\mathfrak{A}_{\zeta}$. In this framework, we prove fusion rules and give statistics operators and a left inverse.

\section{Algebras, Representations and global Endo- morphisms of the Chiral Ising Model}

In this section, we develop and analyze the formalism used by Mack and Schomerus to describe the chiral Ising model.

\subsection{Local $C^{*}$-Algebras and their Universal Algebra}

We begin our investigations with a brief description of the field algebra, the local and the global observable algebras of the chiral Ising model. Our starting point is a Majorana field $\psi$ on the unit circle $S^{1}$ which has anticommutation relations

$$
\left\{\psi(z)^{*}, \psi(w)\right\}=2 \pi \mathrm{i} z \delta(z-w)
$$

and hermiticity condition

$$
\psi(z)^{*}=z \psi(z) .
$$

We consider smeared fields

$$
\psi(f)=\oint_{S^{1}} \frac{d z}{2 \pi \mathrm{i} z^{\frac{1}{2}}} f(z) \psi(z), \quad f(z) \in L^{2}\left(S^{1}\right) .
$$

These objects obey the canonical anticommutation relations (CAR) of the canonical generators of Araki's [1, 2] selfdual CAR-algebra $\mathcal{C}(\mathcal{K}, \Gamma)$ over the Hilbert space $\mathcal{K}=L^{2}\left(S^{1}\right)$ with the antiunitary involution $\Gamma$ of complex conjugation. We have

$$
\left\{\psi(f)^{*}, \psi(g)\right\}=\langle f, g\rangle \mathbf{1}
$$

with

$$
\psi(f)^{*}=\psi(\Gamma f), \quad\langle f, g\rangle=\oint_{S^{1}} \frac{d z}{2 \pi \mathrm{i} z} \overline{f(z)} g(z) .
$$

As local algebras $\mathcal{A}(I)$ with some open interval $I \subset S^{1}, I \neq S^{1}$ we define those unital algebras which are generated by bilinear expressions

$$
B_{I}(f, g)=\psi(f) \psi(g), \quad \operatorname{supp}(f) \subset I, \operatorname{supp}(g) \subset I
$$


in the Majorana fields. These generators are complex linear in both arguments and obey relations

$$
\begin{aligned}
2 B_{I}(f, f) & =\langle\Gamma f, f\rangle \mathbf{1}, \\
2 B_{I}(f, g) B_{I}(g, h) & =\langle\Gamma g, g\rangle B_{I}(f, h), \\
B_{I}(f, g)^{*} & =B_{I}(\Gamma g, \Gamma f),
\end{aligned}
$$

where $f, g, h \in L^{2}\left(S^{1}\right)$ are functions with support in $I$. Next we consider the algebras $\mathcal{A}(I)$ as defined only by these abstract relations. Since the set $\mathcal{J}$ of open, non-void intervals $I \neq S^{1}$ on the circle is not directed there is no inductive limit for the algebras $\mathcal{A}(I), I \in \mathcal{J}$. But with the additional relation

$$
B_{I}(f, g)=B_{J}(f, g), \quad I \subset J
$$

one can construct a global algebra $\mathcal{A}_{\text {univ }}^{C^{*}}$ which is generated by all $B_{I}(f, g)$, $f, g \in L^{2}\left(S^{1}\right)$ and $I \in \mathcal{J}$ 30, 15, 5]. Perhaps one could expect that the result is the even subalgebra of the selfdual CAR algebra over the whole circle $S^{1}$. We will show that this is actually not the case; instead there occurs a central element $Y \in \mathcal{A}_{\text {univ }}^{C^{*}}$ which will finally lead to the fact that $\mathcal{A}_{\text {univ }}^{C^{*}}$ is the direct sum of two of those even CAR algebras. Let now $I_{1}$ and $I_{2}$ be two disjoint intervals and let $J_{+}$and $J_{-}$be intervals containing both of them, one from the left side and one from the right side, so that $J_{+} \cup J_{-}=S^{1}$. Choose real functions $f_{j} \in L^{2}\left(S^{1}\right)$ with $\left\|f_{j}\right\|^{2}=2$ with $\operatorname{supp}\left(f_{j}\right) \subset I_{j}, j=1,2$. Then define

$$
Y=B_{J_{+}}\left(f_{1}, f_{2}\right) B_{J_{-}}\left(f_{2}, f_{1}\right) .
$$

One finds that $Y$ is unitary, self-adjoint and independent of the special choice of $f_{1}, f_{2}, I_{1}, I_{2}, J_{+}, J_{-}$. Moreover, $Y$ is in the center of $\mathcal{A}_{\text {univ }}^{C^{*}}$. For every $\zeta \in S^{1}$ and $I_{\zeta}=S^{1} \backslash\{\zeta\}$, the global algebra $\mathcal{A}_{\text {univ }}^{C^{*}}$ is generated by $\mathcal{A}\left(I_{\zeta}\right)$ and $Y$ 30, 15, 5].

We now want to reconstruct the global, or, "universal" algebra $\mathcal{A}_{\text {univ }}^{C}$ by a global field algebra, the universal Majorana algebra.

Definition 2.1 The universal Majorana algebra Maj is defined as the direct sum of the selfdual $C A R$ algebra over $\left(L^{2}\left(S^{1}\right), \Gamma\right)$ with itself, i.e.

$$
\text { Maj }=\mathcal{C}\left(L^{2}\left(S^{1}\right), \Gamma\right) \oplus \mathcal{C}\left(L^{2}\left(S^{1}\right), \Gamma\right) .
$$

The center of Maj is generated by the element

$$
Y=(-\mathbf{1}) \oplus \mathbf{1}
$$

and we have the two subalgebras

$$
\operatorname{Maj}_{\mathrm{NS}}=\frac{1}{2}(\mathbf{1}-Y) \mathrm{Maj}, \quad \mathrm{Maj}_{\mathrm{R}}=\frac{1}{2}(\mathbf{1}+Y) \mathrm{Maj} .
$$

The universal Majorana algebra is a well defined $C^{*}$-algebra since $\mathcal{C}\left(L^{2}\left(S^{1}\right), \Gamma\right)$ is. For clarifying the connection between our definition and the definition of Maj given by Mack and Schomerus [23] we consider the following two orthonormal bases of $L^{2}\left(S^{1}\right)$

$$
\left\{e_{r}, r \in \mathbb{Z}+\frac{1}{2}\right\} \quad \text { and } \quad\left\{e_{n}, n \in \mathbb{Z}\right\}
$$


where $e_{a}(z)=z^{a}$ for $z=\mathrm{e}^{\mathrm{i} \phi} \in S^{1},-\pi<\phi \leq \pi, a \in \frac{1}{2} \mathbb{Z}$. We define the elements of Maj (Fourier modes)

$$
\begin{array}{ll}
b_{r}=\psi\left(e_{r}\right) \oplus 0, & r \in \mathbb{Z}+\frac{1}{2}, \\
b_{n}=0 \oplus \psi\left(e_{n}\right), & n \in \mathbb{Z} .
\end{array}
$$

Then we have

- $\mathrm{Maj}_{\mathrm{NS}}$ is generated by the modes $b_{r}, r \in \mathbb{Z}+\frac{1}{2}$,

- $\mathrm{Maj}_{\mathrm{R}}$ is generated by the modes $b_{n}, n \in \mathbb{Z}$,

- Maj is generated by the modes $b_{a}, a \in \frac{1}{2} \mathbb{Z}$,

and the Fourier modes satisfy relations

$$
\begin{gathered}
\left\{b_{a}, b_{c}\right\}=\frac{1}{2}\left(\mathbf{1}+(-1)^{2 a} Y\right) \delta_{a,-c}, \quad b_{a}^{*}=b_{-a}, \\
Y b_{a}=(-1)^{2 a} b_{a}, \quad\left[Y, b_{a}\right]=0, \quad Y=Y^{*}, \quad Y^{2}=\mathbf{1} .
\end{gathered}
$$

It is convenient to understand the elements of Maj as smeared fields as well. We define the Hilbert space

$$
\hat{\mathcal{K}}=L^{2}\left(S^{1}\right) \oplus L^{2}\left(S^{1}\right)
$$

which may be identified with $L^{2}\left(\tilde{S}_{1}\right)$, where $\tilde{S}_{1}$ denotes the double cover of $S^{1}$. Hence each element $\hat{f} \in \hat{\mathcal{K}}$ has the unique decomposition

$$
\hat{f}=f_{\mathrm{NS}} \oplus f_{\mathrm{R}}, \quad f_{\mathrm{NS}}, f_{\mathrm{R}} \in L^{2}\left(S^{1}\right) .
$$

On $\hat{\mathcal{K}}$ we have the antiunitary involution

$$
\hat{\Gamma}=\Gamma \oplus \Gamma .
$$

We define the field $\hat{\psi}(\hat{f}) \in$ Maj by

$$
\hat{\psi}(\hat{f})=\psi\left(f_{\mathrm{NS}}\right) \oplus \psi\left(f_{\mathrm{R}}\right),
$$

so that we have the conjugation

$$
\hat{\psi}(\hat{f})^{*}=\hat{\psi}(\hat{\Gamma} \hat{f})
$$

anticommutation relations

$$
\left\{\hat{\psi}(\hat{f})^{*}, \hat{\psi}(\hat{g})\right\}=\frac{1}{2}(\mathbf{1}-Y)\left\langle f_{\mathrm{NS}}, g_{\mathrm{NS}}\right\rangle+\frac{1}{2}(\mathbf{1}+Y)\left\langle f_{\mathrm{R}}, g_{\mathrm{R}}\right\rangle,
$$

boundary condition

$$
Y \hat{\psi}(\hat{f})=\hat{\psi}(y \hat{f}), \quad y=(-\mathbf{1}) \oplus \mathbf{1} \in \mathcal{B}(\hat{\mathcal{K}})
$$

and

$$
[Y, \hat{\psi}(\hat{f})]=0 .
$$

We now want to redefine the local generators $B_{I}(f, g) \in \mathcal{A}(I)$ as even elements of Maj. For that we have to fix an arbitrary point $\zeta \in S^{1}$. We distinguish between two cases: 
Case 1: For all intervals $I \in \mathcal{J}$ with $\zeta \notin I$ we set

$$
B_{I}(f, g)=\hat{\psi}(\hat{f}) \hat{\psi}(\hat{g}), \quad \hat{f}=f \oplus f \in \hat{\mathcal{K}}, \quad \hat{g}=g \oplus g \in \hat{\mathcal{K}} .
$$

Case 2: For every interval $I \in \mathcal{J}$ with $\zeta \in I$ the point $\zeta$ splits $I$ in two disjoint intervals $I_{1}$ and $I_{2}$ so that $I=I_{1} \cup\{\zeta\} \cup I_{2}$. Let $\chi_{j}$ be the characteristic functions of $I_{j}$ and set $f_{j}=\chi_{j} f, g_{j}=\chi_{j} g, j=1,2$. Then we set

$$
\begin{aligned}
B_{I}(f, g)= & \hat{\psi}\left(\hat{f}_{1}\right) \hat{\psi}\left(\hat{g}_{1}\right)+\hat{\psi}\left(\hat{f}_{2}\right) \hat{\psi}\left(\hat{g}_{2}\right)+Y \hat{\psi}\left(\hat{f}_{1}\right) \hat{\psi}\left(\hat{g}_{2}\right)+Y \hat{\psi}\left(\hat{f}_{2}\right) \hat{\psi}\left(\hat{g}_{1}\right), \\
& \hat{f}_{j}=f_{j} \oplus f_{j} \in \hat{\mathcal{K}}, \quad \hat{g}_{j}=g_{j} \oplus g_{j} \in \hat{\mathcal{K}}, \quad j=1,2 .
\end{aligned}
$$

It is an easy but less beautiful work to control that these $B_{I}(f, g)$ satisfy the relations (5) - (9), and that relation (10) is fulfilled with the $Y$ of Definition 2.1, also independent of the functions and intervals [5]. It is not hard to see that the identifications (16), (17) define an isomorphism between $\mathcal{A}_{\text {univ }}^{C^{*}}$ and the even part $\mathrm{Maj}^{+}$of Maj, too 30]. Thus we are allowed to identify the global observable algebra $\mathcal{A}_{\text {univ }}^{C^{*}}$ with the even part of Maj.

\subsection{Representations and Endomorphisms}

Each of the algebras $\mathrm{Maj}_{\mathrm{NS}}$ and $\mathrm{Maj}_{\mathrm{R}}$ possesses a faithful cyclic representation $\left(\mathcal{H}_{\mathrm{NS}}, \pi_{\mathrm{NS}},\left|\Omega_{\mathrm{NS}}\right\rangle\right)$ and $\left(\mathcal{H}_{\mathrm{R}}, \pi_{\mathrm{R}},\left|\Omega_{\mathrm{R}}\right\rangle\right)$ which is characterized by

$$
\begin{aligned}
\pi_{\mathrm{NS}}\left(b_{r}\right)\left|\Omega_{\mathrm{NS}}\right\rangle=0, & r>0, \quad r \in \mathbb{Z}+\frac{1}{2}, \\
\pi_{\mathrm{R}}\left(b_{n}\right)\left|\Omega_{\mathrm{R}}\right\rangle=0, & n>0, \quad n \in \mathbb{Z},
\end{aligned}
$$

respectively. The NS-representation is uniquely characterized (all matrix-elements can be computed and the vector $\left|\Omega_{\mathrm{NS}}\right\rangle$ is defined to be cyclic). In the $\mathrm{R}$-representation, the action of the self-adjoint $b_{0}$ on the cyclic $\left|\Omega_{\mathrm{R}}\right\rangle$ is not completely fixed. To determine the R-representation uniquely too, we require in addition that the vectors $\left|\Omega_{R}\right\rangle$ and $\pi_{R}\left(b_{0}\right)\left|\Omega_{R}\right\rangle$ are orthogonal in $\mathcal{H}_{R}$,

$$
\left\langle\Omega_{\mathrm{R}}\left|\pi_{\mathrm{R}}\left(b_{0}\right)\right| \Omega_{\mathrm{R}}\right\rangle=0 .
$$

One can consider these representations as those of Maj on the space $\mathcal{H}_{\mathrm{NS}} \oplus \mathcal{H}_{\mathrm{R}}$ by the requirement

$$
\pi_{\mathrm{NS}}(Y)=-\mathbf{1}, \quad \pi_{\mathrm{R}}(Y)=\mathbf{1}
$$

which leads automatically to

$$
\pi_{\mathrm{NS}}\left(b_{n}\right)=0, \quad n \in \mathbb{Z} \quad \text { and } \quad \pi_{\mathrm{R}}\left(b_{r}\right)=0, \quad r \in \mathbb{Z}+\frac{1}{2}
$$

i.e. $\pi_{\mathrm{NS}}$ lives only on $\mathrm{Maj}_{\mathrm{NS}}$ and $\pi_{\mathrm{R}}$ on $\mathrm{Maj}_{\mathrm{R}}$. Of course, both representations are then no longer faithful. The NS-representation is irreducible, the R-representation is not; it decomposes into two irreducible subrepresentations $\left(\mathcal{H}_{\mathrm{R}}^{+}, \pi_{\mathrm{R}}^{+}\right)$and $\left(\mathcal{H}_{\mathrm{R}}^{-}, \pi_{\mathrm{R}}^{-}\right)$(see below) which are generated by the action of $\pi_{\mathrm{R}}(\mathrm{Maj})$ on vectors $\left|\Omega_{\mathrm{R}}^{+}\right\rangle$and $\left|\Omega_{\mathrm{R}}^{-}\right\rangle$, respectively, where

$$
\left|\Omega_{\mathrm{R}}^{ \pm}\right\rangle=\frac{1}{\sqrt{2}}\left|\Omega_{\mathrm{R}}\right\rangle \pm \pi_{\mathrm{R}}\left(b_{0}\right)\left|\Omega_{\mathrm{R}}\right\rangle .
$$


These states are eigenstates of $\pi_{\mathrm{R}}\left(b_{0}\right)$ with eigenvalues $\pm 2^{-\frac{1}{2}}$ [29]. We are now interested in what happens, when the representations of Maj, $\pi_{\mathrm{NS}}$ and $\pi_{\mathrm{R}}$, are restricted to the observable algebra which is the even subalgebra of Maj, $\mathcal{A}_{\text {univ }}^{C^{*}}=\mathrm{Maj}^{+}$. It is known that the NS-representation splits into two irreducibles,

$$
\left.\pi_{\mathrm{NS}}\right|_{\mathcal{A}_{\text {univ }}^{C^{*}}}=\pi_{0} \oplus \pi_{1}, \quad \mathcal{H}_{\mathrm{NS}}=\mathcal{H}_{0} \oplus \mathcal{H}_{1},
$$

and the R-representation decomposes into two equivalent ones,

$$
\left.\pi_{\mathrm{R}}\right|_{\mathcal{A}_{\text {univ }}^{C^{*}}}=\pi_{1 / 2} \oplus \pi_{1 / 2}^{\prime}, \quad \mathcal{H}_{\mathrm{R}}=\mathcal{H}_{1 / 2} \oplus \mathcal{H}_{1 / 2}^{\prime} .
$$

The subspaces $\mathcal{H}_{0}, \mathcal{H}_{1}, \mathcal{H}_{1 / 2}$ and $\mathcal{H}_{1 / 2}^{\prime}$ are spanned by vectors

$$
\begin{aligned}
\pi_{\mathrm{NS}}\left(b_{-r_{2 N}} \cdots b_{-r_{1}}\right)\left|\Omega_{\mathrm{NS}}\right\rangle & \in \mathcal{H}_{0}, r_{i} \in \mathbb{N}_{0}+\frac{1}{2}, r_{2 N}>\cdots>r_{1}, \\
\pi_{\mathrm{NS}}\left(b_{-r_{2 N+1}} \cdots b_{-r_{1}}\right)\left|\Omega_{\mathrm{NS}}\right\rangle & \in \mathcal{H}_{1}, r_{i} \in \mathbb{N}_{0}+\frac{1}{2}, r_{2 N+1}>\cdots>r_{1}, \\
\pi_{\mathrm{R}}\left(b_{-n_{2 N}} \cdots b_{-n_{1}}\right)\left|\Omega_{\mathrm{R}}\right\rangle & \in \mathcal{H}_{1 / 2}, \quad n_{i} \in \mathbb{N}_{0}, n_{2 N}>\cdots>n_{1}, \\
\pi_{\mathrm{R}}\left(b_{-n_{2 N+1}} \cdots b_{-n_{1}}\right)\left|\Omega_{\mathrm{R}}\right\rangle & \in \mathcal{H}_{1 / 2}^{\prime}, \quad n_{i} \in \mathbb{N}_{0}, n_{2 N+1}>\cdots>n_{1},
\end{aligned}
$$

with $N \in \mathbb{N}_{0}$. We remark that the subspaces $\mathcal{H}_{1 / 2}$ and $\mathcal{H}_{1 / 2}^{\prime}$ do not coincide with $\mathcal{H}_{\mathrm{R}}^{+}$and $\mathcal{H}_{\mathrm{R}}^{-}$. How is that possible? The reason is that the subrepresentations $\pi_{\mathrm{R}}^{+}$and $\pi_{\mathrm{R}}^{-}$, when restricted to the observable algebra $\mathcal{A}_{\text {univ }}^{C^{*}}$, become equivalent [29], and see below. Therefore the decomposition into invariant subspaces is not unique.

Mack and Schomerus 23, 24] defined the following endomorphisms of Maj which restrict to endomorphisms of the global observable algebra $\mathcal{A}_{\text {univ }}^{C^{*}}$.

Definition 2.2 The endomorphisms $\varrho_{J}, J=0, \frac{1}{2}, 1$ of Maj are defined by their action on the generators as follows,

$$
\begin{gathered}
\varrho_{0}=i d, \\
\varrho_{1 / 2}\left(b_{a}\right)=\left\{\begin{array}{cl}
\mathrm{i} b_{a+\frac{1}{2}} & a \geq \frac{1}{2} \\
\frac{\mathrm{i}}{\sqrt{2}\left(b_{\frac{1}{2}}-b_{-\frac{1}{2}}\right)} & a=0 \\
-\mathrm{i} b_{a-\frac{1}{2}} & a \leq-\frac{1}{2}
\end{array}, \quad \varrho_{1 / 2}(Y)=-Y,\right. \\
\varrho_{1}\left(b_{a}\right)=\left\{\begin{array}{cl}
-b_{a} & a \neq 0, \pm \frac{1}{2} \\
b_{-a} & a=0, \pm \frac{1}{2}
\end{array}, \quad \varrho_{1}(Y)=Y .\right.
\end{gathered}
$$

It is shown [23] that these endomorphisms fulfill

$$
\begin{aligned}
& \pi_{\mathrm{NS}} \circ \varrho_{1 / 2} \cong \pi_{\mathrm{R}}, \\
& \pi_{\mathrm{NS}} \circ \varrho_{1} \cong \pi_{\mathrm{NS}}, \\
& \pi_{0} \circ \varrho_{J} \cong \pi_{J}, \quad J=0, \frac{1}{2}, 1,
\end{aligned}
$$

where relation (29) is the most trivial one because $\varrho_{1}$ is inner in Maj, implemented by the unitary self-adjoint

$$
R=\sqrt{2} b_{0}+b_{\frac{1}{2}}+b_{-\frac{1}{2}} \in \text { Maj. }
$$

We can define these endomorphisms by the formula

$$
\varrho_{J}(\hat{\psi}(\hat{f}))=\hat{\psi}\left(\hat{V}_{J} \hat{f}\right)
$$


where $\hat{V}_{J}$ are the following isometries on $\hat{\mathcal{K}}=L^{2}\left(S^{1}\right) \oplus L^{2}\left(S^{1}\right)$,

$$
\hat{V}_{0}=\left(\begin{array}{cc}
\mathbf{1} & 0 \\
0 & \mathbf{1}
\end{array}\right), \quad \hat{V}_{1 / 2}=\left(\begin{array}{cc}
0 & V_{1 / 2} \\
V_{1 / 2}^{\prime} & 0
\end{array}\right), \quad \hat{V}_{1}=\left(\begin{array}{cc}
V_{1} & 0 \\
0 & V_{1}^{\prime}
\end{array}\right) ;
$$

the isometries (Bogoliubov operators, see below) $V_{1 / 2}, V_{1 / 2}^{\prime}, V_{1}, V_{1}^{\prime} \in \mathcal{B}\left(L^{2}\left(S^{1}\right)\right)$ are defined by

$$
\begin{aligned}
V_{1 / 2} & =\frac{\mathrm{i}}{\sqrt{2}}\left(\left|e_{\frac{1}{2}}\right\rangle\left\langle e_{0}|-| e_{-\frac{1}{2}}\right\rangle\left\langle e_{0}\right|\right)+\mathrm{i} \sum_{n=1}^{\infty}\left(\left|e_{n+\frac{1}{2}}\right\rangle\left\langle e_{n}|-| e_{-n-\frac{1}{2}}\right\rangle\left\langle e_{-n}\right|\right), \\
V_{1 / 2}^{\prime} & =\mathrm{i} \sum_{n=1}^{\infty}\left(\left|e_{n}\right\rangle\left\langle e_{n-\frac{1}{2}}|-| e_{-n}\right\rangle\left\langle e_{-n+\frac{1}{2}}\right|\right), \\
V_{1} & =\left|e_{\frac{1}{2}}\right\rangle\left\langle e_{-\frac{1}{2}}|+| e_{-\frac{1}{2}}\right\rangle\left\langle e_{\frac{1}{2}}\right|-\sum_{n=1}^{\infty}\left(\left|e_{n+\frac{1}{2}}\right\rangle\left\langle e_{n+\frac{1}{2}}|+| e_{-n-\frac{1}{2}}\right\rangle\left\langle e_{-n-\frac{1}{2}}\right|\right), \\
V_{1}^{\prime} & =\left|e_{0}\right\rangle\left\langle e_{0}\right|-\sum_{n=1}^{\infty}\left(\left|e_{n}\right\rangle\left\langle e_{n}|+| e_{-n}\right\rangle\left\langle e_{-n}\right|\right) .
\end{aligned}
$$

It is worthy of note that the two non-vanishing entries, each in $\hat{V}_{1 / 2}$ and $\hat{V}_{1}$, are actually different.

\section{Localized Endomorphisms}

In this section we present our localized endomorphisms in terms of Bogoliubov transformations. After a brief summary of mathematical results which we will use, we introduce them as endomorphisms of the algebra of the punctured circle.

\subsection{The Selfdual CAR Algebra: Some Useful Results}

For a better handling of our techniques we give a brief repetition of Araki's selfdual CAR algebra $\mathcal{C}(\mathcal{K}, \Gamma)$ and quasifree states [1, 2]. We consider a Hilbert space $\mathcal{K}$ with an antiunitary involution $\Gamma$ (complex conjugation), $\Gamma^{2}=\mathbf{1}$, which fulfills

$$
\langle\Gamma f, \Gamma g\rangle=\langle g, f\rangle, \quad f, g \in \mathcal{K} .
$$

The selfdual CAR algebra $\mathcal{C}(\mathcal{K}, \Gamma)$ is defined to be the $C^{*}$-norm closure of the algebra which is generated by the image of a linear mapping $\psi$ which maps elements $f \in \mathcal{K}$ to canonical generators $\psi(f)$, so that

$$
\psi(f)^{*}=\psi(\Gamma f), \quad\left\{\psi(f)^{*}, \psi(g)\right\}=\langle f, g\rangle \mathbf{1}
$$

holds. The $C^{*}$-norm satisfies 2

$$
\|\psi(f)\|=\frac{1}{\sqrt{2}} \sqrt{\|f\|^{2}+\sqrt{\|f\|^{4}-|\langle f, \Gamma f\rangle|^{2}}}
$$

In particular, we have the inequality

$$
\|\psi(f)\| \leq\|f\| .
$$


Elements of the set

$$
\mathcal{I}(\mathcal{K}, \Gamma)=\left\{V \in \mathcal{B}(\mathcal{K}) \mid[V, \Gamma]=0, V^{*} V=\mathbf{1}\right\}
$$

of $\Gamma$ commuting isometries on $\mathcal{K}$ are called Bogoliubov operators. Every Bogoliubov operator $V \in \mathcal{I}(\mathcal{K}, \Gamma)$ defines an endomorphism $\varrho_{V}$ of $\mathcal{C}(\mathcal{K}, \Gamma)$, defined by its action on the canonical generators,

$$
\varrho_{V}(\psi(f))=\psi(V f) .
$$

Moreover, if $V \in \mathcal{I}(\mathcal{K}, \Gamma)$ is surjective (i.e. unitary), then $\varrho_{V}$ is an automorphism.

Definition 3.1 A state $\omega$ of $\mathcal{C}(\mathcal{K}, \Gamma)$ is called quasifree if for all $n \in \mathbb{N}$

$$
\begin{aligned}
\omega\left(\psi\left(f_{1}\right) \cdots \psi\left(f_{2 n+1}\right)\right) & =0 \\
\omega\left(\psi\left(f_{1}\right) \cdots \psi\left(f_{2 n}\right)\right) & =(-1)^{\frac{n(n-1)}{2}} \sum_{\sigma} \operatorname{sign} \sigma \prod_{j=1}^{n} \omega\left(\psi\left(f_{\sigma(j)}\right) \psi\left(f_{\sigma(n+j)}\right)\right)
\end{aligned}
$$

holds. The sum runs over all permutations $\sigma \in \mathcal{S}_{2 n}$ with the property

$$
\sigma(1)<\sigma(2)<\cdots<\sigma(n), \quad \sigma(j)<\sigma(j+n), \quad j=1, \ldots, n .
$$

Quasifree states are therefore completely characterized by their two point function. It is known that there is a one to one correspondence between the set of quasifree states and the set

$$
\mathcal{Q}(\mathcal{K}, \Gamma)=\left\{S \in \mathcal{B}(\mathcal{K}) \mid S=S^{*}, 0 \leq S \leq \mathbf{1}, S+\Gamma S \Gamma=\mathbf{1}\right\},
$$

given by the formula

$$
\omega\left(\psi(f)^{*} \psi(g)\right)=\langle f, S g\rangle .
$$

The quasifree state characterized by Eq. (36) is denoted by $\omega_{S}$. A quasifree state, composed with a Bogoliubov endomorphism is again a quasifree state, namely we have $\omega_{S} \circ \varrho_{V}=\omega_{V * S V}$. The projections in $\mathcal{Q}(\mathcal{K}, \Gamma)$ are called basis projections. If $P$ is a basis projection then the state $\omega_{P}$ is pure and is called a Fock state. The corresponding GNS representation $\left(\mathcal{H}_{P}, \pi_{P},\left|\Omega_{P}\right\rangle\right)$ is irreducible, it is called the Fock representation; the vector $\left|\Omega_{P}\right\rangle \in \mathcal{H}_{P}$ is called the Fock vacuum. Araki proved [1, 2] that a state $\omega$ of $\mathcal{C}(\mathcal{K}, \Gamma)$ which satisfies

$$
\omega\left(\psi(f) \psi(f)^{*}\right)=0, \quad f \in P \mathcal{K}
$$

for a basis projection $P$ is automatically the Fock state $\omega=\omega_{P}$.

We now come to an important quasiequivalence criterion for quasifree states. It was developed for the case of gauge invariant quasifree states by Powers and Størmer [25] and generalized for arbitrary quasifree states by Araki [1]. Unitary equivalence (denoted by "") or quasiequivalence (denoted by " $\approx$ ") of states means always that the corresponding GNS representations are unitarily equivalent or quasiequivalent, respectively.

Theorem 3.2 Two quasifree states $\omega_{S_{1}}$ and $\omega_{S_{2}}$ of $\mathcal{C}(\mathcal{K}, \Gamma)$ are quasiequivalent if and only if

$$
S_{1}^{\frac{1}{2}}-S_{2}^{\frac{1}{2}} \in \mathcal{J}_{2}(\mathcal{K})
$$

where $\mathcal{J}_{2}(\mathcal{K})$ denotes the ideal of Hilbert Schmidt operators in $\mathcal{B}(\mathcal{K})$. 
We now can conclude that two Fock states $\omega_{P_{1}}$ and $\omega_{P_{2}}$ are unitarily equivalent, if and only if $P_{1}-P_{2}$ is Hilbert Schmidt class, or, if $\omega_{P}$ is a Fock state and $\varrho_{V}$ is a Bogoliubov endomorphism, that $\omega_{P} \circ \varrho_{V} \approx \omega_{P}$ if and only if $P-\left(V^{*} P V\right)^{\frac{1}{2}}$ is Hilbert Schmidt class. But in most cases we study representations of the form $\pi_{P} \circ \varrho_{V}$, where $\varrho_{V}$ is a Bogoliubov endomorphism and $\pi_{P}$ a Fock representation of $\mathcal{C}(\mathcal{K}, \Gamma)$. Such a representation $\pi_{P} \circ \varrho_{V}$ is in general not cyclic but it is equivalent to a multiple of the GNS representation $\pi_{V^{*} P V}$ of the state $\omega_{V^{*} P V}=$ $\omega_{P} \circ \varrho_{V}$. The multiplicity is given by $2^{N_{V}}$ where $N_{V}$ is the dimension of the intersection of $\operatorname{ker} V^{*}$ and $P \mathcal{K}$ [ 4 , 27], i.e.

$$
\pi_{P} \circ \varrho_{V} \cong 2^{N_{V}} \pi_{V^{*} P V}, \quad N_{V}=\operatorname{dim}\left(\operatorname{ker} V^{*} \cap P \mathcal{K}\right) .
$$

This is a decomposition of $\pi_{P} \circ \varrho_{V}$ into cyclic subrepresentations but in general not into irreducibles. A decomposition into irreducibles is provided by the following theorem which was proven in [6] and, in a different way, in [4].

Theorem 3.3 Let $V$ be a Bogoliubov operator with $M_{V}=\operatorname{dim} \operatorname{ker} V^{*}<\infty$. If $M_{V}$ is an even integer we have (with notations as above)

$$
\pi_{P} \circ \varrho_{V} \cong 2^{\frac{M_{V}}{2}} \pi_{P^{\prime}}
$$

where $\pi_{P^{\prime}}$ is an (irreducible) Fock representation. If $M_{V}$ is odd then we have

$$
\pi_{P} \circ \varrho_{V} \cong 2^{\frac{M_{V}-1}{2}}\left(\pi_{+} \oplus \pi_{-}\right)
$$

where $\pi_{+}$and $\pi_{-}$are mutually inequivalent, irreducible representations.

The representations $\pi_{ \pm}$occurring in Eq. (41) are called pseudo Fock representations [1]. Consider the automorphism $\alpha_{-1}$ of $\mathcal{C}(\mathcal{K}, \Gamma)$ which is defined by $\alpha_{-1}(\psi(f))=-\psi(f)$. We define the even algebra $\mathcal{C}(\mathcal{K}, \Gamma)^{+}$to be the subalgebra of $\alpha_{-1}$-fixpoints,

$$
\mathcal{C}(\mathcal{K}, \Gamma)^{+}=\left\{x \in \mathcal{C}(\mathcal{K}, \Gamma) \mid \alpha_{-1}(x)=x\right\} .
$$

We now are interested in what happens when our representations of $\mathcal{C}(\mathcal{K}, \Gamma)$ are restricted to the even algebra. For basis projections $P_{1}, P_{2}$, with $P_{1}-P_{2}$ Hilbert Schmidt class, Araki and D.E. Evans [3] defined an index, taking values \pm 1 ,

$$
\operatorname{ind}\left(P_{1}, P_{2}\right)=(-1)^{\operatorname{dim}\left(P_{1} \mathcal{K} \cap\left(\mathbf{1}-P_{2}\right) \mathcal{K}\right)} \text {. }
$$

The automorphism $\alpha_{-1}$ leaves any quasifree state $\omega_{S}$ invariant. Thus $\alpha_{-1}$ is implemented in $\pi_{S}$. In particular, in a Fock representation $\pi_{P}, \alpha_{-1}$ extends to an automorphism $\bar{\alpha}_{-1}$ of $\pi_{P}(\mathcal{C}(\mathcal{K}, \Gamma))^{\prime \prime}=\mathcal{B}\left(\mathcal{H}_{P}\right)$. The following proposition is taken from [2].

Proposition 3.4 Let $U \in \mathcal{I}(\mathcal{K}, \Gamma)$ be a unitary Bogoliubov operator and let $P$ be a basis projection such that $P-U^{*} P U$ is Hilbert-Schmidt class. Denote by $Q(U) \in \mathcal{B}\left(\mathcal{H}_{P}\right)$ the unitary which implements $\varrho_{U}$ in $\pi_{P}$. Then

$$
\bar{\alpha}_{-1}(Q(U))=\sigma(U) Q(U), \quad \sigma(U)= \pm 1 .
$$

In particular,

$$
\sigma(U)=\operatorname{ind}\left(P, U^{*} P U\right)
$$


Furthermore, one has [3, 2]

Theorem 3.5 Restricted to the even algebra $\mathcal{C}(\mathcal{K}, \Gamma)^{+}$, a Fock representation $\pi_{P}$ splits into two mutually inequivalent, irreducible subrepresentations,

$$
\left.\pi_{P}\right|_{\mathcal{C}(\mathcal{K}, \Gamma)^{+}}=\pi_{P}^{+} \oplus \pi_{P}^{-}
$$

Given two basis projections $P_{1}, P_{2}$, then

$$
\pi_{P_{1}}^{ \pm} \cong \pi_{P_{2}}^{ \pm}
$$

if and only if $P_{1}-P_{2} \in \mathcal{J}_{2}(\mathcal{K})$ and $\operatorname{ind}\left(P_{1}, P_{2}\right)=+1$, and

$$
\pi_{P_{1}}^{ \pm} \cong \pi_{P_{2}}^{\mp}
$$

if and only if $P_{1}-P_{2} \in \mathcal{J}_{2}(\mathcal{K})$ and $\operatorname{ind}\left(P_{1}, P_{2}\right)=-1$.

On the other hand, it was proven in [6] that pseudo Fock representations $\pi_{+}$ and $\pi_{-}$of Theorem 3.3, when restricted to the even algebra, remain irreducible but become equivalent. Summarizing we obtain

Theorem 3.6 With notations of Theorem 3.5, a representation $\pi_{P} \circ \varrho_{V}$ restricts as follows to the even algebra $\mathcal{C}(\mathcal{K}, \Gamma)^{+}:$If $M_{V}$ is even we have

$$
\left.\pi_{P} \circ \varrho_{V}\right|_{\mathcal{C}(\mathcal{K}, \Gamma)^{+}} \cong 2^{\frac{M_{V}}{2}}\left(\pi_{P^{\prime}}^{+} \oplus \pi_{P^{\prime}}^{-}\right)
$$

with $\pi_{P^{\prime}}^{+}$and $\pi_{P^{\prime}}^{-}$mutually inequivalent and irreducible. If $M_{V}$ is odd, then

$$
\left.\pi_{P} \circ \varrho_{V}\right|_{\mathcal{C}(\mathcal{K}, \Gamma)^{+}} \cong 2^{\frac{M_{V}+1}{2}} \pi
$$

with $\pi$ irreducible.

\subsection{Restriction to the Algebra of the Punctured Circle}

Let us consider the algebra of the punctured circle $\mathcal{A}\left(I_{\zeta}\right)$. There is no $Y$ in $\mathcal{A}\left(I_{\zeta}\right)$ and the generators are of the form

$$
B_{I_{\zeta}}(f, g)=\hat{\psi}(\hat{f}) \hat{\psi}(\hat{g}), \quad \hat{f}=f \oplus f, \quad \hat{g}=g \oplus g .
$$

Thus we identify $\mathcal{A}\left(I_{\zeta}\right)$ as the even algebra $\mathcal{C}\left(L^{2}\left(S^{1}\right), \Gamma\right)^{+}$and we are allowed to denote the generators by

$$
B_{I}(f, g)=\psi(f) \psi(g), \quad f, g \in L^{2}(I), \quad I \subset I_{\zeta},
$$

i.e. we work with common CAR algebras. By construction (16) and (17) it is easy to see that our representations $\pi_{J}$, being non-faithful on $\mathcal{A}_{\text {univ }}^{C^{*}}$, fulfill

$$
\pi_{J}\left(\mathcal{A}\left(I_{\zeta}\right)\right)=\pi_{J}\left(\mathcal{A}_{\text {univ }}^{C^{*}}\right), \quad J=0, \frac{1}{2}, 1
$$

the representation theories of $\mathcal{A}\left(I_{\zeta}\right)$ and $\mathcal{A}_{\text {univ }}^{C^{*}}$ are obviously the same. Since $\mathcal{A}\left(I_{\zeta}\right) \cong \mathcal{C}\left(L^{2}\left(S^{1}\right), \Gamma\right)^{+}$we can identify representations $\pi_{\mathrm{NS}}$ and $\pi_{\mathrm{R}}$ with GNS 
representations of quasifree states of $\mathcal{C}\left(L^{2}\left(S^{1}\right), \Gamma\right)$ and, correspondingly, representations $\pi_{0}, \pi_{1 / 2}, \pi_{1}$ with associated restrictions to the even subalgebra. This works as follows. Consider $S_{\mathrm{NS}}, S_{\mathrm{R}} \in \mathcal{Q}\left(L^{2}\left(S^{1}\right), \Gamma\right)$, the Neveu Schwarz operator

$$
S_{\mathrm{NS}}=\sum_{r \in \mathbb{N}_{0}+\frac{1}{2}}\left|e_{-r}\right\rangle\left\langle e_{-r}\right|
$$

is a basis projection, the Ramond operator

$$
S_{\mathrm{R}}=\frac{1}{2}\left|e_{0}\right\rangle\left\langle e_{0}\left|+\sum_{n \in \mathbb{N}}\right| e_{-n}\right\rangle\left\langle e_{-n}\right|
$$

is not. By $\left(\mathcal{H}_{S_{\mathrm{NS}}}, \pi_{S_{\mathrm{NS}}},\left|\Omega_{S_{\mathrm{NS}}}\right\rangle\right)$ and $\left(\mathcal{H}_{S_{\mathrm{R}}}, \pi_{S_{\mathrm{R}}},\left|\Omega_{S_{\mathrm{R}}}\right\rangle\right)$ we denote the GNS triples of the corresponding quasifree states $\omega_{S_{\mathrm{NS}}}$ and $\omega_{S_{\mathrm{R}}}$, respectively. We have

$$
\omega_{S_{\mathrm{NS}}}\left(\psi\left(e_{r}\right)^{*} \psi\left(e_{r}\right)\right)=\left\langle e_{r}, S_{\mathrm{NS}} e_{r}\right\rangle=0, \quad r \in \mathbb{N}_{0}+\frac{1}{2}
$$

and therefore, corresponding to Eq. (18),

$$
\pi_{S_{\mathrm{NS}}}\left(\psi\left(e_{r}\right)\right)\left|\Omega_{S_{\mathrm{NS}}}\right\rangle=0, \quad r \in \mathbb{N}_{0}+\frac{1}{2},
$$

as well as

$$
\omega_{S_{\mathrm{R}}}\left(\psi\left(e_{n}\right)^{*} \psi\left(e_{n}\right)\right)=\left\langle e_{n}, S_{\mathrm{R}} e_{n}\right\rangle=0, \quad n \in \mathbb{N}
$$

and therefore, corresponding to Eq. (19),

$$
\pi_{S_{\mathrm{R}}}\left(\psi\left(e_{n}\right)\right)\left|\Omega_{S_{\mathrm{R}}}\right\rangle=0, \quad n \in \mathbb{N} .
$$

Since $\omega_{S_{\mathrm{R}}}$ is quasifree we obtain $\omega_{S_{\mathrm{R}}}\left(\psi\left(e_{0}\right)\right)=0$ as the correspondence to the additional requirement (20). Consider an arbitrary element $x=x_{\mathrm{NS}} \oplus x_{R}$ of Maj, $x_{\mathrm{NS}}, x_{R} \in \mathcal{C}\left(L^{2}\left(S^{1}\right), \Gamma\right)$. By (18), (19) and (22), and taking into consideration that $\pi_{\mathrm{NS}}$ and $\pi_{\mathrm{R}}$ are defined as cyclic representations of Maj, we identify

$$
\pi_{\mathrm{NS}}(x)=\pi_{S_{\mathrm{NS}}}\left(x_{\mathrm{NS}}\right), \quad \pi_{\mathrm{R}}(x)=\pi_{S_{\mathrm{R}}}\left(x_{R}\right) .
$$

Now $\mathcal{A}\left(I_{\zeta}\right)$ is generated by bilinear expressions of Maj with $x_{\mathrm{NS}}=x_{R}$. Therefore, with identification of $\mathcal{A}\left(I_{\zeta}\right)$ and $\mathcal{C}\left(L^{2}\left(S^{1}\right), \Gamma\right)^{+}$, we have to identify $\pi_{\mathrm{NS}}$ with $\pi_{S_{\mathrm{NS}}}, \pi_{\mathrm{R}}$ with $\pi_{S_{\mathrm{R}}}$, and with notations of Theorem 3.5, $\pi_{0}$ with $\pi_{S_{\mathrm{NS}}}^{+}$and $\pi_{1}$ with $\pi_{S_{\mathrm{NS}}}^{-}$. Consider our isometry $V_{1 / 2}$ (the Bogoliubov operator defined at the end of subsection 2.2): The kernel of its adjoint $V_{1 / 2}^{*}$ is one-dimensional, spanned by the vector $2^{-\frac{1}{2}}\left(e_{\frac{1}{2}}+e_{-\frac{1}{2}}\right)$, i.e. $M_{V_{1 / 2}}=1$. By Theorem 3.3 we find $\pi_{S_{\mathrm{NS}}} \circ \varrho_{V_{1 / 2}} \cong \pi_{+} \oplus \pi_{-}$with inequivalent, irreducible pseudo Fock representations $\pi_{ \pm}$, becoming equivalent in the restriction to the even algebra by Theorem 3.6. Since $S_{\mathrm{R}}=V_{1 / 2}^{*} S_{\mathrm{NS}} V_{1 / 2}$ the states $\omega_{S_{\mathrm{R}}}$ and $\omega_{S_{\mathrm{NS}}} \circ \varrho_{V_{1 / 2}}$ coincide. By Eq. (39), $\pi_{S_{N S}} \circ \varrho_{V_{1 / 2}}$ is indeed a GNS representation of $\omega_{\mathrm{R}}$, the Fock vacuum $\left|\Omega_{S_{\mathrm{NS}}}\right\rangle$ is cyclic for $\pi_{S_{\mathrm{NS}}} \circ \varrho_{V_{1 / 2}}$ since $N_{V}=0$. This establishes $\pi_{S_{\mathrm{R}}} \cong \pi_{S_{\mathrm{NS}}} \circ \varrho_{V_{1 / 2}} \cong \pi_{+} \oplus \pi_{-}$. We conclude that the equivalent restrictions of $\pi_{+}$and $\pi_{-}$to the even algebra correspond to the representations $\pi_{1 / 2}$ and $\pi_{1 / 2}^{\prime}$. 


\subsection{Examples of Localized Endomorphisms}

We have seen that, when working on the punctured circle, one has to deal with even CAR algebras. Thus we are allowed to define endomorphisms of $\mathcal{A}\left(I_{\zeta}\right)$ simply as Bogoliubov endomorphisms of the underlying algebra $\mathcal{C}\left(L^{2}\left(S^{1}\right), \Gamma\right)$. We remark that our endomorphisms $\varrho_{1 / 2}$ and $\varrho_{1}$ of $\mathcal{A}_{\text {univ }}^{C^{*}}$ do not restrict to endomorphisms of $\mathcal{A}\left(I_{\zeta}\right)$ because of the different entries $V_{J}$ and $V_{J}^{\prime}$ in matrices $\hat{V}_{J}, J=\frac{1}{2}, 1$ of Eq. (31). For constructing localized endomorphisms, we admit as localization regions all open intervals such that their closure is contained in $I_{\zeta}$, i.e. elements of the set

$$
\mathcal{J}_{\zeta}=\left\{I \in \mathcal{J} \mid \zeta \in I^{\prime}\right\} .
$$

( $I^{\prime}$ always denotes the interior of the complement $I^{c}=S^{1} \backslash I$.) As usual, we define an endomorphism $\varrho$ of $\mathcal{A}\left(I_{\zeta}\right)$ to be localized in some interval $I \in \mathcal{J}_{\zeta}$ if $\varrho(A)=A$ for all $A \in \mathcal{A}\left(I_{1}\right), I_{1} \in \mathcal{J}_{\zeta}, I_{1} \cap I=\emptyset$. We present localized endomorphisms as Bogoliubov endomorphisms which are induced by pseudolocalized isometries. A Bogoliubov operator $V \in \mathcal{I}\left(L^{2}\left(S^{1}\right), \Gamma\right)$ is called pseudolocalized in $I \in \mathcal{J}_{\zeta}$ if for all $f \in L^{2}\left(S^{1}\right)$

$$
(V f)(z)=\sigma_{ \pm} f(z), \quad z \in I_{ \pm}, \quad \sigma_{ \pm} \in\{-1,1\},
$$

where $I_{+}, I_{-}$denote the two connected components of $I^{\prime} \cap I_{\zeta}$. Moreover, $V$ is called even, if $\sigma_{+}=\sigma_{-}$, and odd, if $\sigma_{+}=-\sigma_{-}$. Clearly, a pseudolocalized Bogoliubov operator induces a localized endomorphism of the even algebra $\mathcal{A}\left(I_{\zeta}\right)=\mathcal{C}\left(L^{2}\left(S^{1}\right), \Gamma\right)^{+}$. We give the following examples

Definition 3.7 Let $h \in L^{2}\left(S^{1}\right)$ be a real (i.e. $\Gamma$-invariant) function, $\|h\|=1$ and $\operatorname{supp}(h) \subset I$ for some $I \in \mathcal{J}_{\zeta}$. Define $W \in \mathcal{I}\left(L^{2}\left(S^{1}\right), \Gamma\right)$,

$$
W=2|h\rangle\langle h|-1
$$

and the automorphism $\varrho_{1}^{\text {loc }}=\varrho_{W}$ of $\mathcal{C}\left(L^{2}\left(S^{1}\right), \Gamma\right)$.

Obviously, $W$ is even pseudolocalized and $\varrho_{1}^{\text {loc }}$ therefore, when restricted to $\mathcal{A}\left(I_{\zeta}\right)$, localized in $I$.

Lemma 3.8 The automorphism $\varrho_{1}^{\text {loc }}$ is inner in $\mathcal{C}\left(L^{2}\left(S^{1}\right), \Gamma\right)$. In restriction to the even algebra $\mathcal{A}\left(I_{\zeta}\right)$ it leads to

$$
\pi_{0} \circ \varrho_{1}^{\text {loc }} \cong \pi_{1} .
$$

Proof. One easily checks that $\varrho_{W}$ is implemented in $\mathcal{C}\left(L^{2}\left(S^{1}\right), \Gamma\right)$ by the unitary self-adjoint $q(W)=\sqrt{2} \psi(h)$, for all $f \in L^{2}\left(S^{1}\right)$ we have

$$
\begin{aligned}
q(W) \psi(f) q(W) & =2 \psi(h) \psi(f) \psi(h) \\
& =2\{\psi(h), \psi(f)\} \psi(h)-2 \psi(f) \psi(h) \psi(h) \\
& =2\langle h, f\rangle \psi(h)-\psi(f) \\
& =\psi(2\langle h, f\rangle h-f) \\
& =\psi(W f) .
\end{aligned}
$$

Thus $\varrho_{W}$ is implemented in $\pi_{S_{\mathrm{NS}}}$ by $Q(W)=\pi_{S_{\mathrm{NS}}}(q(W))$. Obviously we have $\bar{\alpha}_{-1}(Q(U))=-Q(U)$ and $S_{\mathrm{NS}}-W^{*} S_{\mathrm{NS}} W$ is Hilbert Schmidt class. By Proposition 3.4 and Theorem 3.5 we conclude

$$
\pi_{S_{\mathrm{NS}}}^{+} \circ \varrho_{W} \cong \pi_{S_{\mathrm{NS}}}^{-}
$$


which proves the lemma, q.e.d.

In the following we are searching for a localized endomorphism $\varrho_{1 / 2}^{\text {loc }}$ which leads to a representation being equivalent to $\pi_{1 / 2}$. It turns out that the discussion becomes much more complicated. First we fix our point $\zeta$ to be $\zeta=-1$, without loss of generality. Further, we choose the localization region $I$ to be $I_{2}$,

$$
I_{2}=\left\{z=\mathrm{e}^{\mathrm{i} \phi} \in S^{1} \mid-\frac{\pi}{2}<\phi<\frac{\pi}{2}\right\}
$$

so that the open complement $I_{2}^{\prime}$ is divided by $\zeta$ into $I_{-}$and $I_{+}$,

$$
\begin{aligned}
& I_{-}=\left\{z=\mathrm{e}^{\mathrm{i} \phi} \in S^{1} \mid-\pi<\phi<-\frac{\pi}{2}\right\}, \\
& I_{+}=\left\{z=\mathrm{e}^{\mathrm{i} \phi} \in S^{1} \mid \frac{\pi}{2}<\phi<\pi\right\} .
\end{aligned}
$$

The Hilbert space $L^{2}\left(S^{1}\right)$ decomposes into a direct sum,

$$
L^{2}\left(S^{1}\right)=L^{2}\left(I_{-}\right) \oplus L^{2}\left(I_{2}\right) \oplus L^{2}\left(I_{+}\right) .
$$

By $P_{I_{+}}, P_{I_{-}}$we denote the projections on the subspaces $L^{2}\left(I_{+}\right), L^{2}\left(I_{-}\right)$, respectively. Define functions on $S^{1}$ by

$$
e_{a}^{(2)}(z)=\left\{\begin{array}{cl}
\sqrt{2} z^{2 a} & z \in I_{2} \\
0 & z \in S^{1} \backslash I_{2}
\end{array} \quad, \quad a \in \frac{1}{2} \mathbb{Z} .\right.
$$

With

$$
\left\{e_{r}^{(2)}, r \in \mathbb{Z}+\frac{1}{2}\right\}, \quad\left\{e_{n}^{(2)}, n \in \mathbb{Z}\right\}
$$

we then obtain two orthonormal bases of the subspace $L^{2}\left(I_{2}\right) \subset L^{2}\left(S^{1}\right)$.

Definition 3.9 We defin@ Bogoliubov operators $V, V^{\prime} \in \mathcal{I}\left(L^{2}\left(S^{1}\right), \Gamma\right)$ as follows,

$$
\begin{aligned}
V= & P_{I_{-}}- \\
& P_{I_{+}}+\frac{\mathrm{i}}{\sqrt{2}}\left(\left|e_{\frac{1}{2}}^{(2)}\right\rangle\left\langle e_{0}^{(2)}|-| e_{-\frac{1}{2}}^{(2)}\right\rangle\left\langle e_{0}^{(2)}\right|\right) \\
& +\mathrm{i} \sum_{n=1}^{\infty}\left(\left|e_{n+\frac{1}{2}}^{(2)}\right\rangle\left\langle e_{n}^{(2)}|-| e_{-n-\frac{1}{2}}^{(2)}\right\rangle\left\langle e_{-n}^{(2)}\right|\right), \\
V^{\prime}= & P_{I_{-}}-P_{I_{+}}+\mathrm{i} \sum_{n=1}^{\infty}\left(\left|e_{n}^{(2)}\right\rangle\left\langle e_{n-\frac{1}{2}}^{(2)}|-| e_{-n}^{(2)}\right\rangle\left\langle e_{-n+\frac{1}{2}}^{(2)}\right|\right) .
\end{aligned}
$$

and let $\varrho_{1 / 2}^{\mathrm{loc}}$ and $\sigma_{1 / 2}^{\mathrm{loc}}$ be the endomorphisms of $\mathcal{C}\left(L^{2}\left(S^{1}\right), \Gamma\right)$ defined by $\varrho_{1 / 2}^{\mathrm{loc}}=\varrho_{V}$ and $\sigma_{1 / 2}^{\text {loc }}=\varrho_{V^{\prime}}$.

Obviously, $V$ and $V^{\prime}$ are odd pseudolocalized and $\varrho_{1 / 2}^{\text {loc }}$ and $\sigma_{1 / 2}^{\text {loc }}$ therefore, when restricted to $\mathcal{A}\left(I_{\zeta}\right)$, localized in $I_{2}$.

\footnotetext{
${ }^{1}$ The definition of $V^{\prime}$ was already suggested by Mack and Schomerus [23].
} 


\subsection{Analysis of $\varrho_{1 / 2}^{\text {loc }}$ and $\sigma_{1 / 2}^{\text {loc }}$}

In this subsection we establish that $\pi_{0} \circ \varrho_{1 / 2}^{\text {loc }}$ is unitarily equivalent to $\pi_{1 / 2}$. Furthermore we identify the unitary equivalence class of $\pi_{0} \circ \varrho_{1 / 2}^{\text {loc }} \varrho_{1 / 2}^{\text {loc }}$. The first step is the following

Lemma 3.10 The following operators are Hilbert Schmidt class,

$$
\begin{aligned}
V^{*} S_{\mathrm{NS}} V-S_{\mathrm{R}} & \in \mathcal{J}_{2}\left(L^{2}\left(S^{1}\right)\right), \\
V S_{\mathrm{NS}} V^{*}-S_{\mathrm{R}} & \in \mathcal{J}_{2}\left(L^{2}\left(S^{1}\right)\right), \\
V^{\prime *} S_{\mathrm{NS}} V^{\prime}-S_{\mathrm{R}} & \in \mathcal{J}_{2}\left(L^{2}\left(S^{1}\right)\right), \\
V^{\prime} S_{\mathrm{NS}} V^{\prime *}-S_{\mathrm{R}} & \in \mathcal{J}_{2}\left(L^{2}\left(S^{1}\right)\right) .
\end{aligned}
$$

Because the proof is ugly work it is banished to the appendix. For drawing our first conclusions of Lemma 3.10, we remember an estimate which was given by Powers and Størmer [25]: For positive operators $A, B \in \mathcal{B}(\mathcal{K})$ the following inequality holds:

$$
\left\|A^{\frac{1}{2}}-B^{\frac{1}{2}}\right\|_{2}^{2} \leq\|A-B\|_{1}
$$

where for $T \in \mathcal{B}\left(L^{2}\left(S^{1}\right)\right)$ by $\|T\|_{1}$ is denoted the trace norm

$$
\|T\|_{1}=\operatorname{tr}\left(\left(T^{*} T\right)^{\frac{1}{2}}\right),
$$

and by $\|T\|_{2}$ the Hilbert Schmidt norm

$$
\|T\|_{2}=\left(\operatorname{tr}\left(T^{*} T\right)\right)^{\frac{1}{2}}
$$

This estimate will be used to see that the operators $V^{*} S_{\mathrm{NS}} V$ and $V^{\prime *} S_{\mathrm{NS}} V^{\prime}$ differ from their positive square roots only by Hilbert Schmidt operators,

$$
\begin{aligned}
\left\|\left(V^{*} S_{\mathrm{NS}} V\right)^{\frac{1}{2}}-V^{*} S_{\mathrm{NS}} V\right\|_{2}^{2} & \leq\left\|V^{*} S_{\mathrm{NS}} V-\left(V^{*} S_{\mathrm{NS}} V\right)^{2}\right\|_{1} \\
& =\left\|V^{*} S_{\mathrm{NS}}\left(\mathbf{1}-V V^{*}\right) S_{\mathrm{NS}} V\right\|_{1} \\
& \leq\|V\|^{2}\left\|S_{\mathrm{NS}}\right\|^{2}\left\|\mathbf{1}-V V^{*}\right\|_{1} .
\end{aligned}
$$

Since $\mathbf{1}-V V^{*}$ is a rank one projection and therefore trace class, the right hand side is finite. Obviously, the same calculation runs for $V^{\prime}$. More easily one finds

$$
\left\|S_{\mathrm{R}}^{\frac{1}{2}}-S_{\mathrm{R}}\right\|_{2}=\|\left(\frac{1}{\sqrt{2}}-\frac{1}{2}\right)\left|e_{0}\right\rangle\left\langle e_{0}\right| \|_{2}=\frac{1}{\sqrt{2}}-\frac{1}{2} .
$$

It follows immediately from Lemma 3.10:

$$
\left(V^{*} S_{\mathrm{NS}} V\right)^{\frac{1}{2}}-S_{\mathrm{R}}^{\frac{1}{2}} \in \mathcal{J}_{2}\left(L^{2}\left(S^{1}\right)\right), \quad\left(V^{\prime *} S_{\mathrm{NS}} V^{\prime}\right)^{\frac{1}{2}}-S_{\mathrm{R}}^{\frac{1}{2}} \in \mathcal{J}_{2}\left(L^{2}\left(S^{1}\right)\right) .
$$

Applying Theorem 3.2 this yields $\omega_{V^{*} S_{\mathrm{NS}} V} \approx \omega_{\mathrm{R}}$ and thus

$$
\pi_{S_{\mathrm{NS}}} \circ \varrho_{V} \approx \pi_{S_{\mathrm{R}}}
$$

the same holds for $\varrho_{V^{\prime}}$. We have already discussed that $\pi_{S_{\mathrm{R}}}$ decomposes into two inequivalent pseudo Fock representations. Using Theorem 3.3, the same is true for $\pi_{S_{\mathrm{NS}}} \circ \varrho_{V}$ and $\pi_{S_{\mathrm{NS}}} \circ \varrho_{V^{\prime}}$ since $M_{V}=M_{V^{\prime}}=1$. Thus we have

$$
\pi_{S_{\mathrm{NS}}} \circ \varrho_{V} \cong \pi_{+} \oplus \pi_{-} \cong \pi_{S_{\mathrm{NS}}} \circ \varrho_{V^{\prime}} .
$$

In restriction to the even algebra $\mathcal{C}\left(L^{2}\left(S^{1}\right), \Gamma\right)^{+}$, the representations $\pi_{+}$and $\pi_{-}$ become equivalent and have to be identified with $\pi_{1 / 2}$. This means $\pi_{S_{\mathrm{NS}}}^{+} \circ \varrho_{V} \cong$ $\pi_{S_{\mathrm{NS}}}^{-} \circ \varrho_{V}$ and $\pi_{S_{\mathrm{NS}}}^{+} \circ \varrho_{V^{\prime}} \cong \pi_{S_{\mathrm{NS}}}^{-} \circ \varrho_{V^{\prime}}$. We have proven 
Theorem 3.11 The representations of $\mathcal{A}\left(I_{\zeta}\right)$ obey

$$
\begin{aligned}
& \pi_{0} \circ \varrho_{1 / 2}^{\mathrm{loc}} \cong \pi_{1} \circ \varrho_{1 / 2}^{\mathrm{loc}} \cong \pi_{1 / 2} \\
& \pi_{0} \circ \sigma_{1 / 2}^{\mathrm{loc}} \cong \pi_{1} \circ \sigma_{1 / 2}^{\mathrm{loc}} \cong \pi_{1 / 2} .
\end{aligned}
$$

Let us now consider the squares $\varrho_{V}^{2}=\varrho_{V^{2}}$ and $\varrho_{V^{\prime}}^{2}=\varrho_{V^{\prime}}$.

Proposition 3.12 The representations of $\mathcal{A}\left(I_{\zeta}\right)$ obey

$$
\begin{aligned}
& \pi_{0} \circ \varrho_{1 / 2}^{\text {loc }} \varrho_{1 / 2}^{\text {loc }} \cong \pi_{0} \oplus \pi_{1} \\
& \pi_{0} \circ \sigma_{1 / 2}^{\text {loc }} \sigma_{1 / 2}^{\text {loc }} \cong \pi_{0} \oplus \pi_{1} .
\end{aligned}
$$

Proof. If we multiply the operator in relation (58) with $V^{*}$ from the left and with $V$ from the right we get

$$
V^{*} S_{\mathrm{R}} V-S_{\mathrm{NS}} \in \mathcal{J}_{2}\left(L^{2}\left(S^{1}\right)\right) .
$$

Since relation (57) holds we can replace $S_{\mathrm{R}}$ by $V^{*} S_{\mathrm{NS}} V$, this yields

$$
V^{*} V^{*} S_{\mathrm{NS}} V V-S_{\mathrm{NS}} \in \mathcal{J}_{2}\left(L^{2}\left(S^{1}\right)\right) .
$$

In the same way one obtains

$$
V^{\prime *} V^{\prime *} S_{\mathrm{NS}} V^{\prime} V^{\prime}-S_{\mathrm{NS}} \in \mathcal{J}_{2}\left(L^{2}\left(S^{1}\right)\right) .
$$

Now the operators $\mathbf{1}-(V V)(V V)^{*}$ and $\mathbf{1}-\left(V^{\prime} V^{\prime}\right)\left(V^{\prime} V^{\prime}\right)^{*}$ are rank two projections, so that we can again conclude

$$
\left(V^{*} V^{*} S_{\mathrm{NS}} V V\right)^{\frac{1}{2}}-S_{\mathrm{NS}} \in \mathcal{J}_{2}\left(L^{2}\left(S^{1}\right)\right) .
$$

and

$$
\left(V^{\prime *} V^{\prime *} S_{\mathrm{NS}} V^{\prime} V^{\prime}\right)^{\frac{1}{2}}-S_{\mathrm{NS}} \in \mathcal{J}_{2}\left(L^{2}\left(S^{1}\right)\right) .
$$

By using Theorem 3.2 we obtain for the states

$$
\omega_{S_{\mathrm{NS}}} \circ \varrho_{V}^{2} \approx \omega_{S_{\mathrm{NS}}}, \quad \omega_{S_{\mathrm{NS}}} \circ \varrho_{V^{\prime}}^{2} \approx \omega_{S_{\mathrm{NS}}} .
$$

Moreover, the kernel of $V^{*}$ is spanned by the $\Gamma$-invariant, normed vector

$$
f_{0}^{(2)}=\frac{1}{\sqrt{2}}\left(e_{\frac{1}{2}}^{(2)}+e_{-\frac{1}{2}}^{(2)}\right) \text {. }
$$

and the kernel of $V^{\prime *}$ is spanned by $e_{0}^{(2)}$. Thus, $\operatorname{ker}\left(V^{*}\right)^{2}\left(\operatorname{resp} . \operatorname{ker}\left(V^{\prime *}\right)^{2}\right)$ is spanned by orthonormal vectors $f_{0}^{(2)}$ and $V f_{0}^{(2)}$ (resp. $e_{0}^{(2)}$ and $\left.V^{\prime} e_{0}^{(2)}\right)$ i.e. $M_{V^{2}}=$ $M_{V^{\prime 2}}=2$; we conclude

$$
\pi_{S_{\mathrm{NS}}} \circ \varrho_{V}^{2} \cong \pi_{S_{\mathrm{NS}}} \oplus \pi_{S_{\mathrm{NS}}} \cong \pi_{S_{\mathrm{NS}}} \circ \varrho_{V^{\prime}}^{2}
$$

by Theorem 3.3. In restriction to the even algebra, identified with $\mathcal{A}\left(I_{\zeta}\right)$, this reads

$$
\begin{aligned}
& \pi_{0} \circ \varrho_{1 / 2}^{\text {loc }} \varrho_{1 / 2}^{\text {loc }} \oplus \pi_{1} \circ \varrho_{1 / 2}^{\text {loc }} \varrho_{1 / 2}^{\text {loc }} \cong \pi_{0} \oplus \pi_{1} \oplus \pi_{0} \oplus \pi_{1} \text {, } \\
& \pi_{0} \circ \sigma_{1 / 2}^{\mathrm{loc}} \sigma_{1 / 2}^{\mathrm{loc}} \oplus \pi_{1} \circ \sigma_{1 / 2}^{\mathrm{loc}} \sigma_{1 / 2}^{\mathrm{loc}} \cong \pi_{0} \oplus \pi_{1} \oplus \pi_{0} \oplus \pi_{1} .
\end{aligned}
$$

We have to assign the irreducible representations on the right hand side to the representations on the left. By Theorem 3.11 we find $\pi_{0} \circ \varrho_{1 / 2}^{\text {loc }} \cong \pi_{1} \circ \varrho_{1 / 2}^{\text {loc }}$ and therefore $\pi_{0} \circ \varrho_{1 / 2}^{\text {loc }} \varrho_{1 / 2}^{\text {loc }} \cong \pi_{1} \circ \varrho_{1 / 2}^{\text {loc }} \varrho_{1 / 2}^{\text {loc }}$. Using the same argument, one obtains $\pi_{0} \circ \sigma_{1 / 2}^{\text {loc }} \sigma_{1 / 2}^{\text {loc }} \cong \pi_{1} \circ \sigma_{1 / 2}^{\text {loc }} \sigma_{1 / 2}^{\text {loc }}$, q.e.d. 


\section{Extension to von Neumann Algebras}

In the DHR theory one usually works with local von Neumann algebras instead of local $C^{*}$-algebras. This formalism allows to discover intertwiners in the observable algebra and this is crucial for the analysis of statistics and fusion. It is our aim to find a description of the chiral Ising model as close as possible to the DHR formalism. Therefore we have to extend our local $C^{*}$-algebras $\mathcal{A}(I)$, to their weak closures in the vacuum representation.

\subsection{The Net of Local von Neumann Algebras}

For intervals $I \in \mathcal{J}$ with non-empty open complement, we define local von Neumann algebras

$$
\mathcal{R}(I)=\pi_{0}(\mathcal{A}(I))^{\prime \prime}, \quad I \in \mathcal{J}, \quad I^{\prime} \neq \emptyset .
$$

By Möbius covariance (some details are presented in the appendix), this defines a so-called covariant precosheaf on the circle. In particular, we have Haag duality on the circle [7, 9],

$$
\mathcal{R}(I)^{\prime}=\mathcal{R}\left(I^{\prime}\right) .
$$

Since the set of intervals $\left\{I \in \mathcal{J}, I^{\prime} \neq \emptyset\right\}$ is not directed we cannot define a global algebra as the $C^{*}$-norm closure of the union of all local algebras. Following Fredenhagen, Rehren and Schroer [14], one could instead introduce the corresponding universal algebra. But in our model it seems to be much more comfortable to define a quasilocal algebra of the punctured circle. (The set $\mathcal{J}_{\zeta}$, Eq. (52), is directed.) This works as follows. We fix again our "point at infinity", without loss of generality, to be $\zeta=-1$ and admit only intervals $I \in \mathcal{J}_{\zeta}$. Then we define our algebra $\mathfrak{A}_{\zeta}$ of quasilocal observables to be the norm closure of all such local von Neumann algebras,

$$
\mathfrak{A}_{\zeta}=\overline{\bigcup_{I \in \mathcal{J}_{\zeta}} \mathcal{R}(I)} .
$$

Now choose some interval $I \in \mathcal{J}_{\zeta}$. Let us denote the von Neumann algebra generated by all $\mathcal{R}\left(I_{0}\right), I_{0} \in \mathcal{J}_{\zeta}, I_{0} \cap I=\emptyset$ by $\mathcal{R}_{\zeta}\left(I^{\prime}\right)$. Obviously we have

$$
\mathcal{R}_{\zeta}\left(I^{\prime}\right) \subset \mathcal{R}\left(I^{\prime}\right) .
$$

We claim that equality holds, that means Haag duality holds also on the punctured circle.

Lemma 4.1 We have Haag duality on the punctured circle. For $I \in \mathcal{J}_{\zeta}$ the following relation holds,

$$
\mathcal{R}(I)^{\prime}=\mathcal{R}_{\zeta}\left(I^{\prime}\right) .
$$

Proof. We have to show $\mathcal{R}_{\zeta}\left(I^{\prime}\right)=\mathcal{R}\left(I^{\prime}\right)$. It is sufficient to show that each generator $\pi_{0}(\psi(f) \psi(g)), \operatorname{supp}(f), \operatorname{supp}(g) \subset I^{\prime}$ of $\mathcal{R}\left(I^{\prime}\right)$ is a weak limit point of a net constructed out of elements in $\mathcal{R}_{\zeta}\left(I^{\prime}\right)$. Let $I_{0} \in \mathcal{J}_{\zeta}, I_{0} \supset I$ be an interval such that $I_{0}^{\prime}$ is a small neighborhood of $\zeta$. Let $\chi_{0}$ be the characteristic function of $I_{0}$ and $f_{0}=\chi_{0} f, g_{0}=\chi_{0} g$. Since $\pi_{0}\left(\psi\left(f_{0}\right) \psi\left(g_{0}\right)\right)$ converges in $C^{*}$-norm to $\pi_{0}(\psi(f) \psi(g))$ by inequality (32) if $I_{0}^{\prime}$ shrinks to the point $\zeta$ it suffices to show 
that $\pi_{0}\left(\psi\left(f_{0}\right) \psi\left(g_{0}\right)\right)$ is such a limit point for all such $I_{0}$. Now let us denote the two connected components of $I^{\prime} \backslash\{\zeta\}$ by $I_{+}$and $I_{-}$. Define $\chi_{ \pm}$to be the characteristic functions of $I_{ \pm}$, and we write $f_{ \pm}=\chi_{ \pm} f_{0}, g_{ \pm}=\chi_{ \pm} g_{0}$. Then

$$
\begin{aligned}
\pi_{0}\left(\psi\left(f_{0}\right) \psi\left(g_{0}\right)\right)= & \pi_{0}\left(\psi\left(f_{+}\right) \psi\left(g_{+}\right)\right)+\pi_{0}\left(\psi\left(f_{-}\right) \psi\left(g_{-}\right)\right) \\
& +\pi_{0}\left(\psi\left(f_{+}\right) \psi\left(g_{-}\right)\right)+\pi_{0}\left(\psi\left(f_{-}\right) \psi\left(g_{+}\right)\right)
\end{aligned}
$$

Clearly, the first two terms on the right hand side are elements of $\mathcal{R}_{\zeta}\left(I^{\prime}\right)$. We show that the third (then, by symmetry, also the fourth) is a weak limit point described as above. Choose sequences $\left\{h_{ \pm}^{(n)}, n \in \mathbb{N}\right\}$, where $h_{ \pm}^{(n)} \in L^{2}\left(S^{1}\right)$ are functions, $\left\|h_{ \pm}^{(n)}\right\|=1, \operatorname{supp}\left(h_{ \pm}^{(n)}\right) \subset I_{ \pm}^{(n)}$, and $I_{ \pm}^{(n)} \in \mathcal{J}_{\zeta}$ are intervals, $I_{ \pm}^{(n)} \subset I_{ \pm} \cap I_{0}^{\prime}$, shrinking to the point $\zeta$. Then define for $n \in \mathbb{N}$

$$
Z_{n}=\pi_{0}\left(\psi\left(h_{+}^{(n)}\right) \psi\left(h_{-}^{(n)}\right)\right) \in \mathcal{R}\left(I^{\prime}\right) .
$$

By Möbius covariance of the vacuum sector (appendix), we can choose the $h_{ \pm}^{(n)}$ such that the $Z_{n}$ are related by Möbius transformations (dilations). For all $n$ we have $\left\|Z_{n}\right\| \leq 1$. It follows that there exists a weakly convergent subnet $\left\{X_{\alpha}, \alpha \in \iota\right\}$, that means there is a function $F: \iota \rightarrow \mathbb{N}(\iota$ a directed set) with the property that $X_{\alpha}=Z_{F(\alpha)}$ for all $\alpha \in \iota$, and that for each $n^{\prime} \in \mathbb{N}$ there is an $\alpha^{\prime} \in \iota$ such that $\alpha \succ \alpha^{\prime}$ implies $F(\alpha) \geq n^{\prime}$ [26]. The weak limit point of the net $\left\{X_{\alpha}, \alpha \in \iota\right\}$ in $\mathcal{R}\left(I^{\prime}\right)$ will be denoted by $X$,

$$
\mathrm{w}-\lim _{\alpha} X_{\alpha}=X \text {. }
$$

For each $I_{1} \in \mathcal{J}_{\zeta}$ all elements $R \in \mathcal{R}\left(I_{1}\right)$ commute with $Z_{n}$ for sufficiently large $n$, thus $[X, R]=0$. It follows

$$
[X, A]=0, \quad A \in \mathfrak{A}_{\zeta},
$$

and, by irreducibility of the vacuum representation, $X$ is a complex number, $X=\lambda \mathbf{1}$. We have

$$
\lambda=\left\langle\Omega_{0}|X| \Omega_{0}\right\rangle=\lim _{\alpha}\left\langle\Omega_{0}\left|X_{\alpha}\right| \Omega_{0}\right\rangle=\lim _{\alpha}\left\langle\Omega_{0}\left|Z_{F(\alpha)}\right| \Omega_{0}\right\rangle=\left\langle\Omega_{0}\left|Z_{1}\right| \Omega_{0}\right\rangle
$$

by Möbius invariance of the vacuum. We claim that we can choose $h_{+}^{(1)}$ and $h_{-}^{(1)}$ such that $\lambda \neq 0$. Recall the definition of the Hardy space

$$
H^{2}=\left\{f \in L^{2}\left(S^{1}\right) \mid\left\langle e_{-n}, f\right\rangle=0, n=1,2, \ldots\right\} .
$$

A Theorem of F. and M. Riesz (see e.g. 12, Th. 6.13) states that

$$
f \in H^{2}, f \neq 0 \quad \Longrightarrow \quad f(z) \neq 0 \text { almost everywhere. }
$$

For example, for a given non-zero function $k \in S_{\mathrm{NS}} L^{2}\left(S^{1}\right)$ we find that $k^{\prime} \in H^{2}$ where $k^{\prime}(z)=z^{\frac{1}{2}} \overline{k(z)}$. So $k^{\prime}$ and hence $k$ cannot vanish on a set of non-zero measure. Now we have

$$
\lambda=\left\langle\Omega_{0}\left|Z_{1}\right| \Omega_{0}\right\rangle=\omega_{0}\left(\psi\left(h_{+}^{(1)}\right) \psi\left(h_{-}^{(1)}\right)\right)=\left\langle\Gamma h_{+}^{(1)}, S_{\mathrm{NS}} h_{-}^{(1)}\right\rangle .
$$


Define $k=S_{\mathrm{NS}} h_{-}^{(1)}$ for a given $h_{-}^{(1)}$ as above. We find $k \neq 0$, otherwise $\Gamma h_{-}^{(1)} \in$ $S_{\mathrm{NS}} L^{2}\left(S^{1}\right)$ in contradiction to the fact that $h_{-}^{(1)}$ and hence $\Gamma h_{-}^{(1)}$ vanishes outside $I_{-}^{(1)}$. It follows $k(z) \neq 0$ almost everywhere and hence

$$
c=\int_{I_{+}^{(1)}}|k(z)|^{2} \frac{d z}{2 \pi \mathrm{i} z} \neq 0
$$

If we define, for instance, $h_{+}^{(1)}(z)=c^{-\frac{1}{2}} \chi_{I_{+}^{(1)}}(z) \overline{k(z)}$ where $\chi_{I_{+}^{(1)}}$ denotes the characteristic function of $I_{+}^{(1)}$ then indeed $\lambda=c^{\frac{1}{2}} \neq 0$. So we can compute

$$
\begin{aligned}
\pi_{0}\left(\psi\left(f_{+}\right) \psi\left(g_{-}\right)\right) & =\lambda^{-1} \pi_{0}\left(\psi\left(f_{+}\right) \psi\left(g_{-}\right)\right) X \\
& =\lambda^{-1} \mathrm{w}-\lim _{\alpha} \pi_{0}\left(\psi\left(f_{+}\right) \psi\left(g_{-}\right)\right) X_{\alpha} \\
& =\lambda^{-1} \mathrm{w}-\lim _{\alpha} \pi_{0}\left(\psi\left(f_{+}\right) \psi\left(g_{-}\right)\right) Z_{F(\alpha)} \\
& =\lambda^{-1} \mathrm{w}-\lim _{\alpha} \pi_{0}\left(\psi\left(f_{+}\right) \psi\left(g_{-}\right) \psi\left(h_{+}^{(F(\alpha))}\right) \psi\left(h_{-}^{(F(\alpha))}\right)\right) \\
& =\lambda^{-1} \mathrm{w}-\lim _{\alpha} \pi_{0}\left(\psi\left(f_{+}\right) \psi\left(h_{+}^{(F(\alpha))}\right) \psi\left(h_{-}^{(F(\alpha))}\right) \psi\left(g_{-}\right)\right) \\
& =\lambda^{-1} \mathrm{w}-\lim _{\alpha} \pi_{0}\left(\psi\left(f_{+}\right) \psi\left(h_{+}^{(F(\alpha))}\right)\right) \pi_{0}\left(\psi\left(h_{-}^{(F(\alpha))}\right) \psi\left(g_{-}\right)\right)
\end{aligned}
$$

i.e. $\pi_{0}\left(\psi\left(f_{+}\right) \psi\left(g_{-}\right)\right)$is indeed a weak limit point of a net of elements in $\mathcal{R}_{\zeta}\left(I^{\prime}\right)$, q.e.d.

\subsection{Extension}

Because we work on the punctured circle the vacuum representation is faithful i.e. $\pi_{0}$ acts faithfully on $\mathcal{A}\left(I_{\zeta}\right)$. Thus we are allowed to use the common convention of identifying observables $A$ with their vacuum representers $\pi_{0}(A)$. Passing over to von Neumann algebras, we consider the vacuum representation acting as the identity on $\mathcal{R}(I)$, and in the same fashion, we treat local $C^{*}$-algebras $\mathcal{A}(I)$ as subalgebras of $\mathcal{R}(I), I \in \mathcal{J}_{\zeta}$. Now we have to check whether we can canonically extend our representations $\pi_{J}$ and endomorphisms $\varrho_{J}^{\text {loc }}, J=\frac{1}{2}, 1$, to the von Neumann algebras $\mathcal{R}(I), I \in \mathcal{J}_{\zeta}$, and the global (quasilocal) $C^{*}$-algebra $\mathfrak{A}_{\zeta}$ they generate. Thus we are looking for isomorphisms

$$
\hat{\pi}_{J}: \mathcal{A}(I)^{\prime \prime}=\mathcal{R}(I) \longrightarrow \pi_{J}(\mathcal{A}(I))^{\prime \prime}
$$

satisfying $\hat{\pi}_{J}(A)=\pi_{J}(A)$ if $A \in \mathcal{A}(I), I \in \mathcal{J}_{\zeta}, J=\frac{1}{2}, 1$. This means exactly that we have to check whether the representations $\pi_{J}$ are quasiequivalent to the identity (vacuum representation) on local algebras $\mathcal{A}(I)$.

Theorem 4.2 (Local Normality) In restriction to local $C^{*}$-algebras $\mathcal{A}(I)$, $I \in \mathcal{J}_{\zeta}$, the representations $\pi_{J}$ are quasiequivalent to the vacuum representation $\pi_{0}=i d$,

$$
\left.\left.\pi_{J}\right|_{\mathcal{A}(I)} \approx \pi_{0}\right|_{\mathcal{A}(I)}, \quad I \in \mathcal{J}_{\zeta}, \quad J=\frac{1}{2}, 1 .
$$

Proof. First we consider the case $J=1$. We have to show that

$$
\left.\left.\pi_{S_{\mathrm{NS}}}^{-}\right|_{\mathcal{C}\left(L^{2}(I), \Gamma\right)^{+}} \approx \pi_{S_{\mathrm{NS}}}^{+}\right|_{\mathcal{C}\left(L^{2}(I), \Gamma\right)^{+}}
$$


We have already proven that $\pi_{S_{\mathrm{NS}}}^{-} \cong \pi_{S_{\mathrm{NS}}}^{+} \circ \varrho_{W}$ on $\mathcal{C}\left(L^{2}\left(S^{1}\right), \Gamma\right)^{+}$. We show that $\pi_{S_{\mathrm{NS}}}^{+} \circ \varrho_{W}$ and $\pi_{S_{\mathrm{NS}}}^{+}$, when restricted to $\mathcal{C}\left(L^{2}(I), \Gamma\right)^{+}$, are unitarily equivalent $\left(I \in \mathcal{J}_{\zeta}\right)$. In $\mathcal{C}\left(L^{2}\left(S^{1}\right), \Gamma\right), \varrho_{W}$ is implemented by the unitary $q(W)=\sqrt{2} \psi(h)$. Choose a real function $h^{\prime} \in L^{2}\left(S^{1}\right)$ such that $\left\|h^{\prime}\right\|=1$ and $\operatorname{supp}\left(h^{\prime}\right) \subset I_{0}$ for some $I_{0} \in \mathcal{J}_{\zeta}, I_{0} \cap I=\emptyset$ and set $U=\sqrt{2} \psi\left(h^{\prime}\right)$. Then $q(W) U$ is a unitary element of $\mathcal{C}\left(L^{2}\left(S^{1}\right), \Gamma\right)^{+}$and for $x \in \mathcal{C}\left(L^{2}(I), \Gamma\right)^{+}$we have

$$
q(W) U x(q(W) U)^{*}=q(W) U x U q(W)=q(W) x q(W)=\varrho_{W}(x)
$$

and therefore

$$
\pi_{S_{\mathrm{NS}}}^{+} \circ \varrho_{W}(x)=\pi_{S_{\mathrm{NS}}}^{+}(q(W) U) \pi_{S_{\mathrm{NS}}}^{+}(x) \pi_{S_{\mathrm{NS}}}^{+}(q(W) U)^{-1}
$$

which proves the statement. Now consider $J=\frac{1}{2}$. By the following Lemma 4.3 we have quasiequivalence of $\pi_{S_{\mathrm{NS}}}$ and $\pi_{S_{\mathrm{R}}}$ on $\mathcal{C}\left(L^{2}(I), \Gamma\right)$. In restriction to the even subalgebra, the irreducible $\pi_{S_{\mathrm{NS}}}$ splits into $\pi_{S_{\mathrm{NS}}}^{+} \oplus \pi_{S_{\mathrm{NS}}}^{-}$, and the two irreducible subrepresentations $\pi_{+}$and $\pi_{-}$of $\pi_{S_{\mathrm{R}}}$ become equivalent to an irreducible representation $\pi$. Thus locally one obtains

$$
\left.\left.\left(\pi_{S_{\mathrm{NS}}}^{+} \oplus \pi_{S_{\mathrm{NS}}}^{-}\right)\right|_{\mathcal{C}\left(L^{2}(I), \Gamma\right)^{+}} \approx 2 \pi\right|_{\mathcal{C}\left(L^{2}(I), \Gamma\right)^{+}}
$$

where $\pi$ corresponds to the representation $\pi_{1 / 2}$. Having already established the local equivalence of $\pi_{S_{\mathrm{NS}}}^{+}$and $\pi_{S_{\mathrm{NS}}}^{-}$this proves the theorem, q.e.d.

Lemma 4.3 For $I \in \mathcal{J}_{\zeta}$ we have the local quasiequivalence

$$
\left.\left.\pi_{S_{\mathrm{NS}}}\right|_{\mathcal{C}\left(L^{2}(I), \Gamma\right)} \approx \pi_{S_{\mathrm{R}}}\right|_{\mathcal{C}\left(L^{2}(I), \Gamma\right)}
$$

Proof. We first claim that $\left|\Omega_{S_{\mathrm{NS}}}\right\rangle$ and $\left|\Omega_{S_{\mathrm{R}}}\right\rangle$ remain cyclic for $\pi_{S_{\mathrm{NS}}}\left(\mathcal{C}\left(L^{2}(I), \Gamma\right)\right)$ and $\pi_{S_{\mathrm{R}}}\left(\mathcal{C}\left(L^{2}(I), \Gamma\right)\right)$, respectively. Denote by $P_{I}$ the projection onto $L^{2}(I) \subset$ $L^{2}\left(S^{1}\right)$. Then for $\pi_{S_{\mathrm{NS}}}$ the statement is a consequence (of the arguments in the proof) of Araki's Lemma 4.8 in [1], because

$$
\left(\mathbf{1}-P_{I}\right) L^{2}\left(S^{1}\right) \cap S_{\mathrm{NS}} L^{2}\left(S^{1}\right)=\{0\} .
$$

(If $k \in S_{\mathrm{NS}} L^{2}\left(S^{1}\right), k \neq 0$, then $k^{\prime}(z)=z^{\frac{1}{2}} \overline{k(z)}$ is a function in $H^{2}$ and hence again by Eq. (59) $k$ and $k^{\prime}$ cannot vanish in the whole interval $I$.) An analogous argument runs for $\pi_{S_{\mathrm{R}}}$, because it is a direct sum of inequivalent pseudo Fock representations $\pi_{+}$and $\pi_{-}$which restrict to Fock representations (see [1] for details) $\pi_{P_{\mathrm{R}}}$ of $\mathcal{C}\left(\left(P_{\mathrm{R}}+\Gamma P_{\mathrm{R}} \Gamma\right) L^{2}\left(S^{1}\right), \Gamma\right)$ each, where

$$
P_{\mathrm{R}}=\sum_{n=1}^{\infty}\left|e_{-n}\right\rangle\left\langle e_{-n}\right|
$$

is a basis projection of $L_{0}^{2}\left(S^{1}\right)=\left(P_{\mathrm{R}}+\Gamma P_{\mathrm{R}} \Gamma\right) L^{2}\left(S^{1}\right)$. If $P_{I}^{(0)}$ denotes the projection onto $L_{0}^{2}(I)=L_{0}^{2}\left(S^{1}\right) \cap L^{2}(I)$ one finds again

$$
\left(\mathbf{1}-P_{I}^{(0)}\right) L_{0}^{2}\left(S^{1}\right) \cap P_{\mathrm{R}} L_{0}^{2}\left(S^{1}\right)=\{0\}
$$

because we have $P_{I}^{(0)}=P_{I}-\left\langle e_{0}, P_{I} e_{0}\right\rangle^{-1} P_{I}\left|e_{0}\right\rangle\left\langle e_{0}\right| P_{I}$ and thus a function $k \in$ $\left(\mathbf{1}-P_{I}^{(0)}\right) L_{0}^{2}\left(S^{1}\right)$ is constant in $I, k(z)=c, z \in I$ and $c \in \mathbb{C}$. On the other 
hand, for $k \in P_{\mathrm{R}} L_{0}^{2}\left(S^{1}\right)$ we find $\Gamma k-\bar{c} \in H^{2}$. Now $\Gamma k-\bar{c}$ vanishes in $I$, so it follows by Eq. (69) that $\Gamma k-\bar{c}=0$, i.e. $k$ is constant on the whole circle, $k=c$. But we have $\left\langle e_{0}, k\right\rangle=0$ for $k \in P_{\mathrm{R}} L_{0}^{2}\left(S^{1}\right)$ and hence $k=0$. So we conclude that the GNS vector $\left|\Omega_{P_{\mathrm{R}}}\right\rangle$ remains cyclic for $\pi_{P_{\mathrm{R}}}\left(\mathcal{C}\left(L_{0}^{2}(I), \Gamma\right)\right)$, thus vectors $\left|\Omega_{ \pm}\right\rangle \equiv\left|\Omega_{P_{\mathrm{R}}}\right\rangle$ for $\pi_{ \pm}\left(\mathcal{C}\left(L^{2}(I), \Gamma\right)\right)$. By inequivalence of $\pi_{+}$and $\pi_{-}$, the GNS vector $\left|\Omega_{S_{\mathrm{R}}}\right\rangle=2^{-\frac{1}{2}}\left(\left|\Omega_{+}\right\rangle \oplus\left|\Omega_{-}\right\rangle\right)$remains cyclic for $\pi_{S_{\mathrm{R}}}\left(\mathcal{C}\left(L^{2}(I), \Gamma\right)\right)$. Thus, for proving the lemma, we have to show that states $\omega_{S_{\mathrm{NS}}}$ and $\omega_{S_{\mathrm{R}}}$ are quasiequivalent on $\mathcal{C}\left(L^{2}(I), \Gamma\right)$. We have to show

$$
\left(P_{I} S_{\mathrm{NS}} P_{I}\right)^{\frac{1}{2}}-\left(P_{I} S_{\mathrm{R}} P_{I}\right)^{\frac{1}{2}} \in \mathcal{J}_{2}\left(L^{2}\left(S^{1}\right)\right) .
$$

Using the inequality (61) it is sufficient to show

$$
\left\|P_{I} S_{\mathrm{NS}} P_{I}-P_{I} S_{\mathrm{R}} P_{I}\right\|_{1}<\infty .
$$

We use the parameterization $z=\mathrm{e}^{\mathrm{i} \phi},-\pi<\phi \leq \pi$ of $S^{1}$. Recall that Hilbert Schmidt operators $A \in \mathcal{J}_{2}\left(L^{2}\left(S^{1}\right)\right)$ can be written as square integrable kernels $A\left(\phi, \phi^{\prime}\right)$. For instance, a rank-one-projection $\left|e_{r}\right\rangle\left\langle e_{r}\right|$ has kernel $\mathrm{e}^{\mathrm{i}\left(\phi-\phi^{\prime}\right)}$. For (small) $\epsilon>0$ define operators in $S_{\mathrm{NS}}^{(\epsilon)}, S_{\mathrm{R}}^{(\epsilon)} \in \mathcal{J}_{2}\left(L^{2}\left(S^{1}\right)\right)$ by kernels

$$
S_{\mathrm{NS}}^{(\epsilon)}\left(\phi, \phi^{\prime}\right)=\sum_{n=0}^{\infty} \mathrm{e}^{-\left(n+\frac{1}{2}\right)\left(\mathrm{i} \phi-\mathrm{i} \phi^{\prime}+\epsilon\right)}=\frac{\mathrm{e}^{-\frac{1}{2}\left(\mathrm{i} \phi-\mathrm{i} \phi^{\prime}+\epsilon\right)}}{1-\mathrm{e}^{-\left(\mathrm{i} \phi-\mathrm{i} \phi^{\prime}+\epsilon\right)}},
$$

and

$$
S_{\mathrm{R}}^{(\epsilon)}\left(\phi, \phi^{\prime}\right)=\frac{1}{2}+\sum_{n=1}^{\infty} \mathrm{e}^{-n\left(\mathrm{i} \phi-\mathrm{i} \phi^{\prime}+\epsilon\right)}=\frac{1}{1-\mathrm{e}^{-\left(\mathrm{i} \phi-\mathrm{i} \phi^{\prime}+\epsilon\right)}}-\frac{1}{2},
$$

Note that $\epsilon$ regularizes the singularities for $\phi-\phi^{\prime}=0, \pm 2 \pi$. Using Cauchy's integral formula, it is easy to check that for $r, s \in \mathbb{Z}+\frac{1}{2}$

$$
\begin{aligned}
\lim _{\epsilon \searrow 0}\left\langle e_{r}, S_{\mathrm{NS}}^{(\epsilon)} e_{s}\right\rangle & =\lim _{\epsilon \searrow 0} \oint_{S^{1}} \frac{d z}{2 \pi \mathrm{i} z} \oint_{S^{1}} \frac{d z^{\prime}}{2 \pi \mathrm{i} z^{\prime}} \frac{z^{-r-\frac{1}{2}} z^{\prime s+\frac{1}{2}}}{1-\frac{z^{\prime}}{z} \mathrm{e}^{-\epsilon}} \\
& =\left\{\begin{array}{cc}
\lim _{\epsilon \searrow 0} \mathrm{e}^{s \epsilon} \delta_{r, s} & r, s<0 \\
0 & \text { otherwise }
\end{array}=\left\langle e_{r}, S_{\mathrm{NS}} e_{s}\right\rangle .\right.
\end{aligned}
$$

Because $\mathrm{e}^{s \epsilon}<1$ for $s<0$ this result can be generalized to

$$
\lim _{\epsilon \searrow 0}\left\langle f, S_{\mathrm{NS}}^{(\epsilon)} g\right\rangle=\left\langle f, S_{\mathrm{NS}} g\right\rangle
$$

for arbitrary $f, g \in L^{2}\left(S^{1}\right)$ by an argument of bounded convergence. So we have weak convergence $\mathrm{w}-\lim _{\epsilon}{ }_{0} S_{\mathrm{NS}}^{(\epsilon)}=S_{\mathrm{NS}}$. In an analogous way one obtains w- $\lim _{\epsilon \searrow_{0}} S_{\mathrm{R}}^{(\epsilon)}=S_{\mathrm{R}}$. Thus the difference $\Delta^{(\epsilon)}=S_{\mathrm{R}}^{(\epsilon)}-S_{\mathrm{NS}}^{(\epsilon)}$ with kernel

$$
\Delta^{(\epsilon)}\left(\phi, \phi^{\prime}\right)=\frac{1}{1+\mathrm{e}^{-\frac{1}{2}\left(\mathrm{i} \phi-\mathrm{i} \phi^{\prime}+\epsilon\right)}}-\frac{1}{2}
$$

converges weakly to $\Delta=S_{\mathrm{R}}-S_{\mathrm{NS}}$. We have to show that $X=P_{I} \Delta P_{I}$ is trace class. The operator $P_{I}$ acts as multiplication with the characteristic function $\chi_{I}(\phi)$ corresponding to $z=\mathrm{e}^{\mathrm{i} \phi} \in I$. Now $X^{(\epsilon)}=P_{I} \Delta^{(\epsilon)} P_{I}$, converging weakly to $X$, has kernel

$$
X^{(\epsilon)}\left(\phi, \phi^{\prime}\right)=\chi_{I}(\phi)\left(\frac{1}{1+\mathrm{e}^{-\frac{1}{2}\left(\mathrm{i} \phi-\mathrm{i} \phi^{\prime}+\epsilon\right)}}-\frac{1}{2}\right) \chi_{I}\left(\phi^{\prime}\right)
$$


and is no more singular for $\epsilon \searrow 0$. Hence

$$
\lim _{\epsilon \searrow 0}\left\langle f, X^{(\epsilon)} g\right\rangle=\int_{-\pi}^{\pi} \frac{d \phi}{2 \pi} \int_{-\pi}^{\pi} \frac{d \phi^{\prime}}{2 \pi} \overline{f\left(\mathrm{e}^{\mathrm{i} \phi}\right)} X^{(\epsilon=0)}\left(\phi, \phi^{\prime}\right) g\left(\mathrm{e}^{\mathrm{i} \phi^{\prime}}\right), \quad f, g \in L^{2}\left(S^{1}\right),
$$

by the theorem of bounded convergence. It follows $X=X^{(\epsilon=0)} \in \mathcal{J}_{2}\left(L^{2}\left(S^{1}\right)\right)$. Let $\tilde{\chi}_{I}$ be a smooth function on $[-\pi, \pi]$ which satisfies $\tilde{\chi}_{I}(\phi)=1$ for $z=\mathrm{e}^{\mathrm{i} \phi} \in I$ and vanishes in a neighborhood of $\phi= \pm \pi$. We define

$$
\tilde{X}\left(\phi, \phi^{\prime}\right)=\tilde{\chi}_{I}(\phi)\left(\frac{1}{1+\mathrm{e}^{-\frac{i}{2}\left(\phi-\phi^{\prime}\right)}}-\frac{1}{2}\right) \tilde{\chi}_{I}\left(\phi^{\prime}\right)
$$

such that $X=P_{I} \tilde{X} P_{I}$ and hence

$$
\|X\|_{1}=\left\|P_{I} \tilde{X} P_{I}\right\|_{1} \leq\left\|P_{I}\right\|\|\tilde{X}\|_{1}\left\|P_{I}\right\|=\|\tilde{X}\|_{1} .
$$

Since $\tilde{X}\left(\phi, \phi^{\prime}\right)$ is a smooth function in $\phi$ and $\phi^{\prime}$ it has fast decreasing Fourier coefficients which coincide with matrix elements $\left\langle e_{n}, \tilde{X} e_{m}\right\rangle, n, m \in \mathbb{Z}$. This proves the statement $\|X\|_{1} \leq \infty$, q.e.d.

We have proven local quasiequivalence of our representations $\pi_{0}, \pi_{1 / 2}$ and $\pi_{1}$. Thus we have an extension to local von Neumann algebras $\mathcal{R}(I)$ and to the quasilocal algebra $\mathfrak{A}_{\zeta}$ they generate. By unitary equivalence $\varrho_{J}^{\text {loc }} \cong \pi_{J}$ on $\mathcal{A}\left(I_{\zeta}\right)$ i.e. there are unitaries $U_{J}: \mathcal{H}_{0} \rightarrow \mathcal{H}_{J}$ satisfying $\varrho_{J}^{\text {loc }}(A)=U_{J}^{-1} \pi_{J}(A) U_{J}$ for all $A \in \mathcal{A}(I)$ and all $I \in \mathcal{J}_{\zeta}$, we have an extension of $\varrho_{J}^{\text {loc }}$ to $\mathfrak{A}_{\zeta}$, too, $J=\frac{1}{2}, 1$. These extensions, denoted by the same symbols, fulfill

$$
\varrho_{J}^{\mathrm{loc}}(A)=A, \quad A \in \mathcal{R}\left(I_{1}\right), \quad I_{1} \in \mathcal{J}_{\zeta}, \quad I_{1} \cap I=\emptyset
$$

( $I$ denotes the localization region) and also

$$
\varrho_{J}^{\mathrm{loc}}\left(\mathcal{R}\left(I_{0}\right)\right) \subset \mathcal{R}\left(I_{0}\right) ; \quad I_{0} \in \mathcal{J}_{\zeta}, \quad I \subset I_{0},
$$

because these endomorphisms satisfy the corresponding relations on the underlying $C^{*}$-algebras $\mathcal{A}\left(I_{1}\right)$ and $\mathcal{A}\left(I_{0}\right)$. We have established that our endomorphisms $\varrho_{J}^{\text {loc }}, J=\frac{1}{2}, 1$, are well-defined localized endomorphisms in the common sense. In addition, these endomorphisms are transportable. This follows because the precosheaf $\{\mathcal{R}(I)\}$ is Möbius covariant. Thus $\mathfrak{A}_{\zeta}$ is covariant with respect to the subgroup of Möbius transformations leaving $\zeta$, the point at infinity, invariant.

\subsection{Fusion Rules of Localized Endomorphisms}

The main advantage of working with local von Neumann algebras is that one can manifestly prove fusion rules in terms of equivalence classes of localized endomorphisms. Let $\varrho_{a}$ and $\varrho_{b}$ be endomorphisms of $\mathfrak{A}_{\zeta}$ localized in intervals $I_{a}, I_{b} \in \mathcal{J}_{\zeta}$, respectively. Then there is an interval $I \in \mathcal{J}_{\zeta}, I_{a} \cup I_{b} \subset I$, such that $\varrho_{a}$ and $\varrho_{b}$ are localized in $I$. Suppose that $\varrho_{a} \cong \varrho_{b}$, i.e. that there is a unitary $U \in \mathcal{B}\left(\mathcal{H}_{0}\right)$ such that $\varrho_{a}(A)=U^{*} \varrho_{b}(A) U$ for all $A \in \mathfrak{A}_{\zeta}$. Exploiting Haag duality one finds $U \in \mathcal{R}(I)$, i.e. $U \in \mathfrak{A}_{\zeta}$. This is an important tool which enables to derive fusion rules in the algebraic framework: If also $\tilde{\varrho}_{a}(A)=\tilde{U}^{*} \tilde{\varrho}_{b}(A) \tilde{U}$, $A \in \mathfrak{A}_{\zeta}$, for localized endomorphisms $\tilde{\varrho}_{a}, \tilde{\varrho}_{b}$ then $\tilde{\varrho}_{a} \varrho_{a} \cong \tilde{\varrho}_{b} \varrho_{b}$, realized by the well-defined unitary $\tilde{\varrho}_{b}(U) \tilde{U}$. So we can deduce fusion rules in terms of equivalence classes by computing it for some special representatives. Obviously, 
this procedure fails for global endomorphisms $\varrho_{J}, J=\frac{1}{2}, 1$, of $\mathcal{A}_{\text {univ }}^{C^{*}}$ by two reasons: The first one is that $\mathcal{A}_{\text {univ }}^{C^{*}}$ is generated by local $C^{*}$-algebras. But local intertwiners may lie only in their weak closures; there is a rather small number of endomorphisms which are inner equivalent in the $C^{*}$-algebras. The second reason is that the vacuum representation does not act faithfully on $\mathcal{A}_{\text {univ }}^{C^{*}}$. In the vacuum representation, too much information gets lost. For example, the equivalence class of $\pi_{0} \circ \varrho_{1 / 2}$ does not depend on the isometry $V_{1 / 2}^{\prime}$ at all but the representation $\pi_{0} \circ \varrho_{1 / 2}^{2}$ does. Counterexamples can be constructed; there is, for instance, an endomorphism $\mu_{1 / 2}$ such that $\pi_{0} \circ \mu_{1 / 2} \cong \pi_{0} \circ \varrho_{1 / 2}$, but $\pi_{0} \circ \mu_{1 / 2}^{2} \not \pi_{0} \circ \varrho_{1 / 2}^{2}$ as representations of $\mathcal{A}_{\text {univ }}^{C^{*}}$ [5].

It is no problem to compute the fusion rules for our special examples of localized endomorphisms, we just have to summarize some of our previous results. Proposition 3.12 gives us the first fusion rule, we have established

$$
\pi_{0} \circ \varrho_{1 / 2}^{\text {loc }} \varrho_{1 / 2}^{\text {loc }} \cong \pi_{0} \oplus \pi_{1} .
$$

We obtain the second fusion rule by the fact that $\pi_{1} \circ \varrho_{1 / 2}^{\text {loc }} \cong \pi_{1 / 2}$ (Theorem 3.11). Hence we conclude

$$
\pi_{0} \circ \varrho_{1}^{\mathrm{loc}} \varrho_{1 / 2}^{\mathrm{loc}} \cong \pi_{1} \circ \varrho_{1 / 2}^{\mathrm{loc}} \cong \pi_{1 / 2} .
$$

Since $\varrho_{1}^{\text {loc }}$ and $\varrho_{1 / 2}^{\text {loc }}$ commute if we choose the localization region of $\varrho_{1}^{\text {loc }}$ disjoint to that of $\varrho_{1 / 2}^{\text {loc }}$ we also obtain

$$
\pi_{0} \circ \varrho_{1 / 2}^{\text {loc }} \varrho_{1}^{\text {loc }} \cong \pi_{1 / 2} .
$$

Trivially, the fact that $\left(\varrho_{1}^{\text {loc }}\right)^{2}=i d$ leads us to the third fusion rule

$$
\pi_{0} \circ \varrho_{1}^{\text {loc }} \varrho_{1}^{\text {loc }} \cong \pi_{0} .
$$

Denoting by $\varrho_{0}$ the identity endomorphism (everywhere localized) and by $\left[\varrho_{J}\right]$ the equivalence class of localized endomorphisms being unitarily equivalent to $\varrho_{J}^{\text {loc }}$ in the vacuum representation, we summarize

\section{Theorem 4.4 (Fusion rules of localized endomorphisms)}

$$
\begin{aligned}
{\left[\varrho_{1 / 2}^{2}\right] } & =\left[\varrho_{0}\right]+\left[\varrho_{1}\right], \\
{\left[\varrho_{1 / 2} \varrho_{1}\right]=\left[\varrho_{1} \varrho_{1 / 2}\right] } & =\left[\varrho_{1 / 2}\right], \\
{\left[\varrho_{1}^{2}\right] } & =\left[\varrho_{0}\right],
\end{aligned}
$$

i.e. the localized endomorphisms obey the Ising fusion rules.

\subsection{Statistics Operator and Left Inverse}

According to the general theory of superselection sectors [11, 13, 17], we expect that for each endomorphism $\varrho$ which is localized in some interval $I \in \mathcal{J}_{\zeta}$ there exists a unitary $\varepsilon_{\varrho} \in \mathcal{R}(I)$ which commutes with $\varrho^{2}\left(\mathfrak{A}_{\zeta}\right)$,

$$
\varepsilon_{\varrho} \in \varrho^{2}\left(\mathfrak{A}_{\zeta}\right)^{\prime}
$$

and fulfills

$$
\varepsilon_{\varrho} \varrho\left(\varepsilon_{\varrho}\right) \varepsilon_{\varrho}=\varrho\left(\varepsilon_{\varrho}\right) \varepsilon_{\varrho} \varrho\left(\varepsilon_{\varrho}\right) .
$$


Therefore the elements $\tau_{i}=\varrho^{i-1}\left(\varepsilon_{\varrho}\right), i=1,2, \ldots$, satisfy the Artin relations and determine a representation of the braid group $B_{\infty}[11,13]$. The statistics operator is given by the formula

$$
\varepsilon_{\varrho}=U^{-1} \varrho(U)
$$

where $U$ is unitary such that the (equivalent) endomorphism $\tilde{\varrho}$, defined by

$$
\tilde{\varrho}(A)=U \varrho(A) U^{-1}, \quad A \in \mathfrak{A}_{\zeta}
$$

is localized in some interval $I_{0} \in \mathcal{J}_{\zeta}, I_{0} \subset I^{\prime}$. The statistics operator is independent of the special choice of $\tilde{\varrho}$ as far as $I_{0}$ varies in one of the two connected components of $I^{\prime} \backslash\{\zeta\}$ but it may depend on the fact whether $I_{0}$ lies in the left or the right complement of $I$ with respect to our "point at infinity" $\zeta$. The computation of $\varepsilon_{\varrho}$ is straightforward for $\varrho=\varrho_{1}^{\text {loc }}$. Let $\varrho$ be induced by a real function $h \in L^{2}\left(S^{1}\right)$ with support in some interval $I,\|h\|^{2}=1$, as described in Definition 3.7. Analogously, let $\tilde{\varrho}$ be induced by a real function $h_{0},\left\|h_{0}\right\|=1$, with $\operatorname{supp}\left(h_{0}\right) \subset I_{0}, I_{0} \cap I=\emptyset$. Since these endomorphisms are unitarily implemented we find

$$
U=2 \psi\left(h_{0}\right) \psi(h), \quad U^{-1}=2 \psi(h) \psi\left(h_{0}\right)
$$

and

$$
\varrho(U)=2 \psi\left(-h_{0}\right) \psi(h)=-U
$$

so that

$$
\varepsilon_{\varrho}=-1
$$

expressing nothing but anticommutativity of Majorana fields. We now want to construct the statistics operator $\varepsilon_{\sigma}$ for our localized endomorphism $\sigma=\sigma_{1 / 2}^{\text {loc }}$. It seems to be very difficult to do that by the formula $(76)$ but to be much easier to determine it by its properties. The statistics operator commutes with $\sigma^{2}\left(\mathfrak{A}_{\zeta}\right)$. The commutant $\sigma^{2}\left(\mathfrak{A}_{\zeta}\right)^{\prime}$ is spanned by elements $1, \Pi$ where the projection $\Pi$ is defined by

$$
\Pi=\psi\left(e_{+}\right) \psi\left(e_{-}\right), \quad e_{ \pm}=\frac{1}{\sqrt{2}}\left(e_{0}^{(2)} \pm \mathrm{i} V^{\prime} e_{0}^{(2)}\right) .
$$

(We remark that the orthonormal vectors $e_{ \pm}$span the kernel of $\left(V^{\prime} V^{\prime}\right)^{*}$ and satisfy $e_{+}=\Gamma e_{-}$.) This leads us to the ansatz

$$
\varepsilon_{\sigma}=\alpha(\mathbf{1}+\gamma \Pi), \quad \alpha, \gamma \in \mathbb{C} .
$$

Now $\varepsilon_{\sigma}$ is unitary,

$$
\varepsilon_{\sigma} \varepsilon_{\sigma}^{*}=|\alpha|^{2}(\mathbf{1}+(\gamma+\bar{\gamma}+\gamma \bar{\gamma}) \Pi)=\mathbf{1} .
$$

Therefore $\gamma+\bar{\gamma}+\gamma \bar{\gamma}=0,|\alpha|^{2}=1$, we write $\alpha=\mathrm{e}^{\mathrm{i} \omega}, \omega$ real. The statistics operator satisfies Eq. (75); we exclude the case $\gamma=0$ and find

$$
\begin{aligned}
0 & =\mathrm{e}^{-3 \mathrm{i} \omega} \gamma^{-1}\left(\varepsilon_{\sigma} \sigma\left(\varepsilon_{\sigma}\right) \varepsilon_{\sigma}-\sigma\left(\varepsilon_{\sigma}\right) \varepsilon_{\sigma} \sigma\left(\varepsilon_{\sigma}\right)\right) \\
& =(\gamma+1)(\Pi-\sigma(\Pi))+\gamma^{2}(\Pi \sigma(\Pi) \Pi-\sigma(\Pi) \Pi \sigma(\Pi)) .
\end{aligned}
$$

It is not hard to see that $\Pi$ and $\sigma(\Pi)$ can be written in the following way:

$$
\Pi=\frac{1}{2}\left(\mathbf{1}+2 \mathrm{i} \psi\left(V^{\prime} e_{0}^{(2)}\right) \psi\left(e_{0}^{(2)}\right)\right), \quad \sigma(\Pi)=\frac{1}{2}\left(\mathbf{1}+2 \mathrm{i} \psi\left(\left(V^{\prime}\right)^{2} e_{0}^{(2)}\right) \psi\left(V^{\prime} e_{0}^{(2)}\right)\right) .
$$


The fields obey

$$
\psi\left(e_{0}^{(2)}\right)^{2}=\psi\left(V^{\prime} e_{0}^{(2)}\right)^{2}=\psi\left(\left(V^{\prime}\right)^{2} e_{0}^{(2)}\right)^{2}=\frac{1}{2} \mathbf{1},
$$

and

$$
\left\{\psi\left(V^{\prime} e_{0}^{(2)}\right), \psi\left(e_{0}^{(2)}\right)\right\}=\left\{\psi\left(\left(V^{\prime}\right)^{2} e_{0}^{(2)}\right), \psi\left(e_{0}^{(2)}\right)\right\}=\left\{\psi\left(\left(V^{\prime}\right)^{2} e_{0}^{(2)}\right), \psi\left(V^{\prime} e_{0}^{(2)}\right)\right\}=0 .
$$

Using these relations one finds?

$$
\Pi \sigma(\Pi) \Pi=\frac{1}{2} \Pi, \quad \sigma(\Pi) \Pi \sigma(\Pi)=\frac{1}{2} \sigma(\Pi)
$$

so that we obtain

$$
\left(\gamma+1+\frac{1}{2} \gamma^{2}\right)(\Pi-\sigma(\Pi))=0 .
$$

Since $\Pi-\sigma(\Pi) \neq 0$ we have

$$
\gamma^{2}+2 \gamma+2=0 \quad \Longleftrightarrow \quad \gamma=-1 \pm \mathrm{i}
$$

and therefore

$$
\varepsilon_{\sigma}=\mathrm{e}^{\mathrm{i} \omega}(\mathbf{1}-(1 \pm i) \Pi) .
$$

According to the general theory [11, 13, 17, we expect that there exists also a left inverse $\Phi_{\sigma}$ to our endomorphism $\sigma$ such that $\Phi_{\sigma} \circ \sigma=i d$. The left inverse is a unital, positive mapping from $\mathfrak{A}_{\zeta}$ to $\mathfrak{A}_{\zeta}$ which satisfies $\Phi_{\sigma}(\mathcal{R}(I)) \subset \mathcal{R}(I)$ if $I \supset I_{2}$. Since $\sigma$ is not an automorphism $\Phi_{\sigma}$ does in general not respect products but

$$
\Phi_{\sigma}(\sigma(A) B \sigma(C))=A \Phi_{\sigma}(B) C
$$

holds for $A, B, C \in \mathfrak{A}_{\zeta}$. In the following we want to derive an explicit description for $\Phi_{\sigma}$. We introduce an arbitrary orthonormal basis $\left\{v_{n}, n \in \mathbb{Z}\right\}$ of $L^{2}\left(S^{1}\right)$ with $v_{0}=e_{0}^{(2)}$ and $\Gamma v_{n}=v_{-n}$. It suffices to consider elements $A$ of $\mathfrak{A}_{\zeta}$ which are sums of monomials $X$ of the form

$$
X=\psi\left(v_{n_{1}}\right) \psi\left(v_{n_{2}}\right) \cdots \psi\left(v_{n_{2 K}}\right) .
$$

Using the anticommutation relations,

$$
\left\{\psi\left(v_{n}\right), \psi\left(v_{n^{\prime}}\right)\right\}=\delta_{n,-n^{\prime}} \mathbf{1},
$$

in particular $\psi\left(v_{0}\right)^{2}=\frac{1}{2} \mathbf{1}$, we can write every monomial $X$ such that $\psi\left(v_{0}\right)$ appears at most once. If every monomial $X$ is written in that way we define $\Phi_{\sigma}$ as the linear mapping which preserves the unit and fulfills

$$
\Phi_{\sigma}(X)=\psi\left(V^{\prime *} v_{n_{1}}\right) \psi\left(V^{\prime *} v_{n_{2}}\right) \cdots \psi\left(V^{\prime *} v_{n_{2 K}}\right) .
$$

It is no problem to check that $\Phi_{\sigma}$ is well defined and has the required properties. The general theory says

$$
\Phi_{\sigma}\left(\varepsilon_{\sigma}\right)=\frac{\omega_{\sigma}}{d(\sigma)} \mathbf{1}
$$

\footnotetext{
${ }^{2}$ With the identification $E_{n}=\sigma^{n-1}(\Pi), n=1,2, \ldots$, this is nothing else but the Temperley-Lieb-Jones algebra relation$$
E_{n} E_{n \pm 1} E_{n}=d(\sigma)^{-2} E_{n}
$$

with statistical dimension $d(\sigma)=\sqrt{2}$.
} 
where $\omega_{\sigma}$ is a phase factor ("statistical phase") and the positive real number $d(\sigma)$ is called statistical dimension. Since $\sigma=\sigma_{1 / 2}^{\text {loc }}$ belongs to the sector $\left[\varrho_{1 / 2}\right]$ we expect that $d(\sigma)=\sqrt{2}$. Using our formula for $\Phi_{\sigma}$ we find (respecting that $V^{\prime} e_{0}^{(2)}$ is orthogonal to $\left.e_{0}^{(2)}\right)$

$$
\Phi_{\sigma}(\Pi)=\Phi_{\sigma}\left(\frac{1}{2}\left(\mathbf{1}+2 \mathrm{i} \psi\left(V^{\prime} e_{0}^{(2)}\right) \psi\left(e_{0}^{(2)}\right)\right)\right)=\frac{1}{2} \mathbf{1} .
$$

We conclude

$$
\Phi_{\sigma}\left(\varepsilon_{\sigma}\right)=\mathrm{e}^{\mathrm{i} \omega}\left(1-\left(\frac{1}{2} \pm \frac{\mathrm{i}}{2}\right)\right) \mathbf{1}=\frac{\mathrm{e}^{\mathrm{i}\left(\omega \mp \frac{\pi}{4}\right)}}{\sqrt{2}} \mathbf{1},
$$

in agreement with $d(\sigma)=\sqrt{2}$. At the end we find

$$
\varepsilon_{\sigma}=\frac{\omega_{\sigma}}{\sqrt{2}}((1 \pm \mathrm{i}) \mathbf{1} \mp 2 \mathrm{i} \Pi) \text {. }
$$

By the spin and statistics theorem [14] we expect that the statistical phase is given by $\omega_{\sigma}=\mathrm{e}^{2 \pi \mathrm{i} s}$ where $s$ is the infimum of the spectrum of the conformal energy operator $L_{0}$ in the representation $\pi_{0} \circ \sigma$. Since $\sigma$ belongs to the sector $\left[\varrho_{1 / 2}\right]$ we have $s=\frac{1}{16}$ and therefore $\omega_{\sigma}=\mathrm{e}^{\frac{\mathrm{i} \pi}{8}}$. However, we did not succeed in computing $\omega_{\sigma}$ directly. Moreover, we observe the freedom to choose the \pm -sign in our formula for the statistics operator $\varepsilon_{\sigma}$. The change of this sign corresponds to the replacement of $\varepsilon_{\sigma}$ by $\varepsilon_{\sigma}^{*}$. The fact that $\varepsilon_{\sigma} \neq \varepsilon_{\sigma}^{*}$ goes back to the nontrivial spacetime topology which is the origin of braid statistics. At the end we remark that the same calculations we have done for $\sigma=\sigma_{1 / 2}^{\text {loc }}$ run for the endomorphism $\varrho_{1 / 2}^{\text {loc }}$; we just have to replace $V^{\prime}$ by $V$ and $e_{0}^{(2)}$ by $f_{0}^{(2)}$.

\section{A Appendix: Möbius Covariance of the Vac- uum Sector}

We will briefly discuss Möbius covariance here. Related topics can be found in the book of Lang [20]. The Möbius symmetry on the circle $S^{1}$ is given by the group $\mathrm{Mob}=\mathrm{SU}(1,1) / \mathbb{Z}_{2}$ where

$$
\mathrm{SU}(1,1)=\left\{g=\left.\left(\begin{array}{cc}
\alpha & \beta \\
\bar{\beta} & \bar{\alpha}
\end{array}\right) \in \mathrm{GL}_{2}(\mathbb{C})|| \alpha\right|^{2}-|\beta|^{2}=1\right\} .
$$

Its action on the circle is

$$
g z=\frac{\bar{\alpha} z-\bar{\beta}}{-\beta z+\alpha}, \quad z \in S^{1} .
$$

Each element $g \in \mathrm{SU}(1,1)$ can be decomposed in the product of a rotation $r(t)$ and a transformation $g^{\prime}=r(t)^{-1} g$ leaving the point $z=-1$ invariant,

$$
g=r(t) g^{\prime}, \quad r(t)=\left(\begin{array}{cc}
\mathrm{e}^{-\frac{\mathrm{i} t}{2}} & 0 \\
0 & \mathrm{e}^{\mathrm{i} t} \frac{1}{2}
\end{array}\right), \quad t \in \mathbb{R}, \quad g^{\prime}=\left(\begin{array}{cc}
\alpha^{\prime} & \frac{\beta^{\prime}}{\beta^{\prime}}
\end{array}\right),
$$

such that $\left(\overline{\alpha^{\prime}}+\overline{\beta^{\prime}}\right)\left(\alpha^{\prime}+\beta^{\prime}\right)^{-1}=1$. Since $r(t+2 \pi)=-r(t)$ we can determine $-2 \pi<t \leq 2 \pi$ uniquely by the additional requirement $\operatorname{Re}\left(\alpha^{\prime}\right)>0$. Then a 
representation $U$ of $\mathrm{SU}(1,1)$ in our Hilbert space of test functions $L^{2}\left(S^{1}\right)$ is defined by

$$
(U(g) f)(z)=\epsilon(g ; z)(\alpha+\bar{\beta} \bar{z})^{-\frac{1}{2}}(\bar{\alpha}+\beta z)^{-\frac{1}{2}} f\left(\frac{\alpha z+\bar{\beta}}{\beta z+\bar{\alpha}}\right)
$$

where for $z=\mathrm{e}^{\mathrm{i} \phi},-\pi<\phi \leq \pi$,

$$
\epsilon(g ; z)=-\operatorname{sign}(t-\pi-\phi) \operatorname{sign}(t+\pi-\phi),
$$

and $\operatorname{sign}(\mathrm{x})=1$ if $x \geq 0, \operatorname{sign}(x)=-1$ if $x<0$. We observe that $\epsilon(g ; z)$ is discontinuous at $z=-1$ and $z=g(-1)=-(\bar{\alpha}+\bar{\beta})(\alpha+\beta)^{-1}$. Up to this $\epsilon$-factor, Eq. (78) is a well-known definition of a representation of $\mathrm{SU}(1,1)$. So it remains to be checked that

$$
\epsilon\left(g_{1} ; z\right) \epsilon\left(g_{2} ; g_{1}^{-1} z\right)=\epsilon\left(g_{1} g_{2} ; z\right) .
$$

Since both sides have their discontinuities at $z=-1$ and $z=g_{1} g_{2}(-1)$ they can differ only by a global sign. But this possibility is easily excluded by arguments of $L^{2}$-continuity in $g$. Moreover, by computing $\left\langle U(g) e_{r}, U(g) e_{s}\right\rangle=\delta_{r, s}$ for $r, s \in \mathbb{Z}+\frac{1}{2}$ (NS-base) we can also check that $U$ is unitary,

$$
\begin{aligned}
\left\langle U(g) e_{r}, U(g) e_{s}\right\rangle & =\oint_{S^{1}} \frac{d z}{2 \pi \mathrm{i} z}(\alpha+\bar{\beta} \bar{z})^{-1}(\bar{\alpha}+\beta z)^{-1}\left(\frac{\alpha z+\bar{\beta}}{\beta z+\bar{\alpha}}\right)^{s-r} \\
& =\frac{1}{2 \pi \mathrm{i}} \oint_{S^{1}} d z(\alpha z+\bar{\beta})^{s-r-1}(\beta z+\bar{\alpha})^{r-s-1} \\
& =\left\{\begin{array}{cc}
0 & s>r \\
\left.\frac{\alpha^{s-r-1}}{(r-s) !} \frac{d^{r-s}}{d z^{r-s}}(\beta z+\bar{\alpha})^{r-s-1}\right|_{z=-\frac{\bar{\beta}}{\alpha}} & s \leq r \\
& =\delta_{r, s}
\end{array}\right.
\end{aligned}
$$

by Cauchy's integral formula, respecting that $|\alpha|^{2}>|\beta|^{2}$ since $|\alpha|^{2}-|\beta|^{2}=$ 1. Since the prefactor on the right hand side in Eq. (78) is real we observe $[U(g), \Gamma]=0$ and hence each $U(g), g \in \mathrm{SU}(1,1)$ induces a Bogoliubov automorphism $\alpha_{g}=\varrho_{U(g)}$ of $\mathcal{C}\left(L^{2}\left(S^{1}\right), \Gamma\right)$. Hence $\mathrm{SU}(1,1)$ is represented by automorphisms of $\mathcal{C}\left(L^{2}\left(S^{1}\right), \Gamma\right)$, and this restricts to a representation of Mob by automorphisms of $\mathcal{C}\left(L^{2}\left(S^{1}\right), \Gamma\right)^{+}$. In order to establish Möbius invariance of the vacuum state and hence covariance of the vacuum sector we show that

$$
\left[S_{\mathrm{NS}}, U(g)\right]=0, \quad g \in \mathrm{SU}(1,1),
$$

i.e. that $U(g)$ respects the polarization of $L^{2}\left(S^{1}\right)$ induced by $S_{\mathrm{NS}}$. It is sufficient to show that

$$
\left\langle e_{-r}, U(g) e_{s}\right\rangle=0, \quad r, s \in \mathbb{N}_{0}+\frac{1}{2}, \quad g \in \mathrm{SU}(1,1) .
$$

The functions $e_{r}(z), r \in \mathbb{Z}+\frac{1}{2}$ are smooth on $S^{1}$ except at their cut at $z=-1$. The prefactor $\epsilon(g ; z)$ in Eq. (78) achieves that $\left(U(g) e_{r}\right)(z)$ remains a smooth function except at $z=-1$, i.e. that the cut is not transported to $g(-1)$. Hence we have

$$
\left(U(g) e_{r}\right)(z)= \pm(\alpha+\bar{\beta} \bar{z})^{-\frac{1}{2}}(\bar{\alpha}+\beta z)^{-\frac{1}{2}}\left(\frac{\alpha z+\bar{\beta}}{\beta z+\bar{\alpha}}\right)^{r}
$$


where all the half-odd integer powers are to be taken in the same branch with cut at $z=-1$. So we can compute as follows,

$$
\begin{aligned}
\left\langle e_{-r}, U(g) e_{s}\right\rangle & = \pm \oint_{S^{1}} \frac{d z}{2 \pi \mathrm{i} z} z^{r}(\alpha z+\bar{\beta})^{-\frac{1}{2}} z^{\frac{1}{2}}(\bar{\alpha}+\beta z)^{-\frac{1}{2}}\left(\frac{\alpha z+\bar{\beta}}{\beta z+\bar{\alpha}}\right)^{s} \\
& = \pm \frac{1}{2 \pi \mathrm{i}} \oint_{S^{1}} d z z^{r-\frac{1}{2}}(\alpha z+\bar{\beta})^{s-\frac{1}{2}}(\bar{\alpha}+\beta z)^{-s-\frac{1}{2}}=0,
\end{aligned}
$$

again by Cauchy's formula, respecting $|\alpha|^{2}>|\beta|^{2}$ and that $r, s$ are positive half-odd integers here.

\section{B Appendix: The Proof of Lemma 5.3}

An essential fact we use for the proof of Lemma 3.10 is presented in the following

Lemma B.1 The difference of the two odd pseudolocalized Bogoliubov operators, given in Definition 3.9 is Hilbert Schmidt class,

$$
V-V^{\prime} \in \mathcal{J}_{2}\left(L^{2}\left(S^{1}\right)\right) .
$$

Proof. Since $\mathbf{1}-P_{0}^{(2)}$ is Hilbert Schmidt class, where

$$
P_{0}^{(2)}=\mathbf{1}-\left|e_{0}^{(2)}\right\rangle\left\langle e_{0}^{(2)}\right|=P_{I_{+}}+P_{I_{-}}+\sum_{n=1}^{\infty}\left(\left|e_{n}^{(2)}\right\rangle\left\langle e_{n}^{(2)}|+| e_{-n}^{(2)}\right\rangle\left\langle e_{-n}^{(2)}\right|\right),
$$

it is equivalent to prove

$$
\Sigma_{0}=\left\|P_{0}^{(2)}\left(V-V^{\prime}\right) P_{0}^{(2)}\right\|_{2}^{2}<\infty .
$$

We remember that the square of the Hilbert Schmidt norm is the sum over the squares of all matrix elements in any Hilbert space basis. Obviously, the Bogoliubov operators $V$ and $V^{\prime}$ differ only on the subspace $L^{2}\left(I_{2}\right) \subset L^{2}\left(S^{1}\right)$. We compute

$$
\begin{aligned}
\Sigma_{0}= & \sum_{\substack{n \in \mathbb{Z} \\
n \neq 0}} \sum_{\substack{m \in \mathbb{Z} \\
m \neq 0}}\left|\left\langle e_{n}^{(2)}, V e_{m}^{(2)}\right\rangle-\left\langle e_{n}^{(2)}, V^{\prime} e_{m}^{(2)}\right\rangle\right|^{2} \\
= & \sum_{n=1}^{\infty} \sum_{m=1}^{\infty}\left|\mathrm{i}\left\langle e_{n}^{(2)}, e_{m+\frac{1}{2}}^{(2)}\right\rangle-\mathrm{i}\left\langle e_{n-\frac{1}{2}}^{(2)}, e_{m}^{(2)}\right\rangle\right|^{2} \\
& +\sum_{n=1}^{\infty} \sum_{m=-1}^{-\infty}\left|-\mathrm{i}\left\langle e_{n}^{(2)}, e_{m-\frac{1}{2}}^{(2)}\right\rangle-\mathrm{i}\left\langle e_{n-\frac{1}{2}}^{(2)}, e_{m}^{(2)}\right\rangle\right|^{2} \\
& +\sum_{n=-1}^{-\infty} \sum_{m=1}^{\infty}\left|\mathrm{i}\left\langle e_{n}^{(2)}, e_{m+\frac{1}{2}}^{(2)}\right\rangle+\mathrm{i}\left\langle e_{n+\frac{1}{2}}^{(2)}, e_{m}^{(2)}\right\rangle\right|^{2} \\
& +\sum_{n=-1}^{-\infty} \sum_{m=-1}^{-\infty}\left|-\mathrm{i}\left\langle e_{n}^{(2)}, e_{m-\frac{1}{2}}^{(2)}\right\rangle+\mathrm{i}\left\langle e_{n+\frac{1}{2}}^{(2)}, e_{m}^{(2)}\right\rangle\right|^{2} .
\end{aligned}
$$


Since $\left\langle e_{n \pm \frac{1}{2}}^{(2)}, e_{m}^{(2)}\right\rangle=\left\langle e_{n}^{(2)}, e_{m \mp \frac{1}{2}}^{(2)}\right\rangle$ the first and the fourth summation vanishes, so that one finds by substituting to positive summation indices

$$
\begin{aligned}
\Sigma_{0}= & \sum_{n=1}^{\infty} \sum_{m=1}^{\infty}\left|\left\langle e_{n}^{(2)}, e_{-m-\frac{1}{2}}^{(2)}\right\rangle+\left\langle e_{n-\frac{1}{2}}^{(2)}, e_{-m}^{(2)}\right\rangle\right|^{2} \\
& +\sum_{n=1}^{\infty} \sum_{m=1}^{\infty}\left|\left\langle e_{-n}^{(2)}, e_{m+\frac{1}{2}}^{(2)}\right\rangle+\left\langle e_{-n+\frac{1}{2}}^{(2)}, e_{m}^{(2)}\right\rangle\right|^{2} \\
= & 2 \sum_{n=1}^{\infty} \sum_{m=1}^{\infty}\left|\left\langle e_{-n}^{(2)}, e_{m+\frac{1}{2}}^{(2)}\right\rangle+\left\langle e_{-n+\frac{1}{2}}^{(2)}, e_{m}^{(2)}\right\rangle\right|^{2},
\end{aligned}
$$

we used $\left\langle e_{-n+\frac{1}{2}}^{(2)}, e_{m}^{(2)}\right\rangle=\overline{\left\langle e_{n-\frac{1}{2}}^{(2)}, e_{-m}^{(2)}\right\rangle}$. The remaining matrix elements are easily computed,

$$
\begin{aligned}
\left\langle e_{-n}^{(2)}, e_{m+\frac{1}{2}}^{(2)}\right\rangle & =2 \int_{-\frac{\pi}{2}}^{\frac{\pi}{2}} \mathrm{e}^{2 \mathrm{i}\left(m+n+\frac{1}{2}\right) \phi} \frac{d \phi}{2 \pi}=+\frac{(-1)^{m+n}}{\pi} \frac{1}{m+n+\frac{1}{2}}, \\
\left\langle e_{-n+\frac{1}{2}}^{(2)}, e_{m}^{(2)}\right\rangle & =2 \int_{-\frac{\pi}{2}}^{\frac{\pi}{2}} \mathrm{e}^{2 \mathrm{i}\left(m+n-\frac{1}{2}\right) \phi} \frac{d \phi}{2 \pi}=-\frac{(-1)^{m+n}}{\pi} \frac{1}{m+n-\frac{1}{2}} .
\end{aligned}
$$

It follows

$$
\Sigma_{0}=\frac{2}{\pi^{2}} \sum_{n=1}^{\infty} \sum_{m=1}^{\infty} \frac{1}{\left((n+m)^{2}-\frac{1}{4}\right)^{2}}=\frac{2}{\pi^{2}} \sum_{k=1}^{\infty} \frac{k}{\left((k+1)^{2}-\frac{1}{4}\right)^{2}}<\infty, \quad \text { q.e.d. }
$$

Now we can start proving Lemma 3.10. We introduce the following notations

$$
\begin{aligned}
B & =V^{*} S_{\mathrm{NS}} V-S_{\mathrm{R}}, \\
P_{0} & =\left|e_{-1}\right\rangle\left\langle e_{-1}|+| e_{0}\right\rangle\left\langle e_{0}|+| e_{1}\right\rangle\left\langle e_{1}\right|, \\
P_{1} & =\sum_{n=1}^{\infty}\left|e_{-2 n-1}\right\rangle\left\langle e_{-2 n-1}\right|, \\
P_{2} & =\sum_{n=1}^{\infty}\left|e_{2 n+1}\right\rangle\left\langle e_{2 n+1}\right|, \\
P_{3} & =\sum_{n=1}^{\infty}\left|e_{-2 n}\right\rangle\left\langle e_{-2 n}\right|, \\
P_{4} & =\sum_{n=1}^{\infty}\left|e_{2 n}\right\rangle\left\langle e_{2 n}\right|,
\end{aligned}
$$

such that we find

$$
\sum_{i=0}^{4} P_{i}=\mathbf{1}, \quad \Gamma P_{1}=P_{2} \Gamma, \quad \Gamma P_{3}=P_{4} \Gamma .
$$

At first we have to show, that $\|B\|_{2}<\infty$. Since $P_{0}$ is Hilbert Schmidt class it is equivalent to prove that

$$
\left\|\left(\mathbf{1}-P_{0}\right) B\left(\mathbf{1}-P_{0}\right)\right\|_{2}=\left\|\sum_{i, j=1}^{4} P_{i} B P_{j}\right\|_{2} \leq \sum_{i, j=1}^{4}\left\|P_{i} B P_{j}\right\|_{2}<\infty .
$$


This will be done by estimating each term $\left\|P_{i} B P_{j}\right\|_{2}$ for its own. Since $B=B^{*}$ we find

$$
\left\|P_{i} B P_{j}\right\|_{2}=\left\|\left(P_{i} B P_{j}\right)^{*}\right\|_{2}=\left\|P_{j} B P_{i}\right\|_{2},
$$

so that we are allowed to treat only those ten of sixteen terms with $i \leq j$. Further, by

$$
\Gamma B \Gamma=V^{*} \Gamma S_{\mathrm{NS}} \Gamma V-\Gamma S_{\mathrm{R}} \Gamma=V^{*}\left(\mathbf{1}-S_{\mathrm{NS}}\right) V-\left(\mathbf{1}-S_{\mathrm{R}}\right)=-B
$$

we find the identity

$$
\left\|P_{1} B P_{1}\right\|_{2}=\left\|\Gamma P_{1} B P_{1} \Gamma\right\|_{2}=\left\|P_{2} \Gamma B \Gamma P_{2}\right\|_{2}=\left\|P_{2} B P_{2}\right\|_{2},
$$

and in the same way

$$
\left\|P_{3} B P_{3}\right\|_{2}=\left\|P_{4} B P_{4}\right\|_{2}, \quad\left\|P_{2} B P_{3}\right\|_{2}=\left\|P_{1} B P_{4}\right\|_{2}, \quad\left\|P_{1} B P_{3}\right\|_{2}=\left\|P_{2} B P_{4}\right\|_{2} .
$$

In each term on the right hand side one of the projections $P_{2}$ or $P_{4}$ appears, but since

$$
P_{2} S_{\mathrm{R}}=S_{\mathrm{R}} P_{2}=P_{4} S_{\mathrm{R}}=S_{\mathrm{R}} P_{4}=0
$$

we have only to prove the finiteness of the six norms

$$
\begin{array}{lll}
\left\|P_{2} V^{*} S_{\mathrm{NS}} V P_{2}\right\|_{2}, & \left\|P_{1} V^{*} S_{\mathrm{NS}} V P_{2}\right\|_{2}, & \left\|P_{1} V^{*} S_{\mathrm{NS}} V P_{4}\right\|_{2}, \\
\left\|P_{2} V^{*} S_{\mathrm{NS}} V P_{4}\right\|_{2}, & \left\|P_{3} V^{*} S_{\mathrm{NS}} V P_{4}\right\|_{2}, & \left\|P_{4} V^{*} S_{\mathrm{NS}} V P_{4}\right\|_{2},
\end{array}
$$

and, since $V-V^{\prime}$ is Hilbert Schmidt class, this is equivalent to prove the finiteness of

$$
\begin{array}{r}
\left\|P_{2} V^{\prime *} S_{\mathrm{NS}} V^{\prime} P_{2}\right\|_{2}, \quad\left\|P_{1} V^{\prime *} S_{\mathrm{NS}} V^{\prime} P_{2}\right\|_{2}, \quad\left\|P_{1} V^{\prime *} S_{\mathrm{NS}} V P_{4}\right\|_{2}, \\
\left\|P_{2} V^{\prime *} S_{\mathrm{NS}} V P_{4}\right\|_{2}, \quad\left\|P_{3} V^{*} S_{\mathrm{NS}} V P_{4}\right\|_{2}, \quad\left\|P_{4} V^{*} S_{\mathrm{NS}} V P_{4}\right\|_{2} .
\end{array}
$$

At first we consider

$$
\begin{aligned}
\Sigma_{1} & =\left\|P_{2} V^{\prime *} S_{\mathrm{NS}} V^{\prime} P_{2}\right\|_{2}^{2}=\sum_{n=1}^{\infty} \sum_{m=1}^{\infty}\left|\left\langle e_{2 n+1}, V^{\prime *} S_{\mathrm{NS}} V^{\prime} e_{2 m+1}\right\rangle\right|^{2} \\
& =\sum_{n=1}^{\infty} \sum_{m=1}^{\infty}\left|\sum_{r \in \mathbb{N}_{0}+\frac{1}{2}} \overline{\left\langle e_{-r}, V^{\prime} e_{2 n+1}\right\rangle}\left\langle e_{-r}, V^{\prime} e_{2 m+1}\right\rangle\right|^{2} .
\end{aligned}
$$

Since $\left\langle e_{m+\frac{1}{2}}^{(2)}, e_{2 n+1}\right\rangle=2^{-\frac{1}{2}} \delta_{n, m}$ the action of $V^{\prime}$ on odd basis vectors $e_{2 n+1}$ is simple, one reads by definition

$$
\left(V^{\prime} e_{2 n+1}\right)(z)=\left\{\begin{array}{cc}
e_{2 n+1}(z) & z \in I_{-} \\
\mathrm{i} e_{2 n+2}(z) & z \in I_{2} \\
-e_{2 n+1}(z) & z \in I_{+}
\end{array} \quad n \in \mathbb{N} .\right.
$$

This leads us to

$$
\begin{aligned}
& \left\langle e_{-r}, V^{\prime} e_{2 n+1}\right\rangle= \\
& =\int_{-\pi}^{-\frac{\pi}{2}} \mathrm{e}^{\mathrm{i}(2 n+1+r) \phi} \frac{d \phi}{2 \pi}+\mathrm{i} \int_{-\frac{\pi}{2}}^{\frac{\pi}{2}} \mathrm{e}^{\mathrm{i}(2 n+2+r) \phi} \frac{d \phi}{2 \pi}-\int_{\frac{\pi}{2}}^{\pi} \mathrm{e}^{\mathrm{i}(2 n+1+r) \phi} \frac{d \phi}{2 \pi} \\
& =\frac{\mathrm{i}(-1)^{n}}{\pi} \sin \left(\frac{r \pi}{2}\right) \frac{1}{(2 n+1+r)(2 n+2+r)} .
\end{aligned}
$$


Substituting to integer summation indices we obtain

$$
\Sigma_{1}=\frac{64}{\pi^{4}} \sum_{n=1}^{\infty} \sum_{m=1}^{\infty}\left(\sum_{l=0}^{\infty} \sigma_{n, m, l}^{(1)}\right)^{2}
$$

where

$$
\sigma_{n, m, l}^{(1)}=\frac{1}{(4 n+2 l+3)(4 n+2 l+5)(4 m+2 l+3)(4 m+2 l+5)} .
$$

We put off the estimate of this summation for some time and pass over to the next sum.

$$
\Sigma_{2}=\left\|P_{1} V^{\prime *} S_{\mathrm{NS}} V^{\prime} P_{2}\right\|_{2}^{2}=\sum_{n=1}^{\infty} \sum_{m=1}^{\infty}\left|\sum_{r \in \mathbb{N}_{0}+\frac{1}{2}} \overline{\left\langle e_{-r}, V^{\prime} e_{-2 n-1}\right\rangle}\left\langle e_{-r}, V^{\prime} e_{2 m+1}\right\rangle\right|^{2} .
$$

The action of $V^{\prime}$ on vectors $e_{-2 n-1}$ is

$$
\left(V^{\prime} e_{-2 n-1}\right)(z)=\left\{\begin{array}{cl}
e_{-2 n-1}(z) & z \in I_{-} \\
-\mathrm{i} e_{-2 n-2}(z) & z \in I_{2} \\
-e_{-2 n-1}(z) & z \in I_{+}
\end{array} \quad n \in \mathbb{N} .\right.
$$

This leads us to

$$
\left\langle e_{-r}, V e_{-2 n-1}\right\rangle=\frac{\mathrm{i}(-1)^{n}}{\pi} \sin \left(\frac{r \pi}{2}\right) \frac{1}{(2 n+1-r)(2 n+2-r)},
$$

so that

$$
\Sigma_{2}=\frac{64}{\pi^{4}} \sum_{n=1}^{\infty} \sum_{m=1}^{\infty}\left(\sum_{l=0}^{\infty} \sigma_{n, m, l}^{(2)}\right)^{2}
$$

where

$$
\sigma_{n, m, l}^{(2)}=\frac{1}{(4 n-2 l+1)(4 n-2 l+3)(4 m+2 l+3)(4 m+2 l+5)} .
$$

Analogously,

$$
\Sigma_{3}=\left\|P_{1} V^{\prime *} S_{\mathrm{NS}} V P_{4}\right\|_{2}^{2}=\sum_{n=1}^{\infty} \sum_{m=1}^{\infty}\left|\sum_{r \in \mathbb{N}_{0}+\frac{1}{2}} \overline{\left\langle e_{-r}, V^{\prime} e_{-2 n-1}\right\rangle}\left\langle e_{-r}, V e_{2 m}\right\rangle\right|^{2} .
$$

The action of $V$ on basis vectors $e_{2 m}$ is

$$
\left(V e_{2 n}\right)(z)=\left\{\begin{array}{cl}
e_{2 n}(z) & z \in I_{-} \\
\mathrm{i} e_{2 n+1}(z) & z \in I_{2} \\
-e_{2 n}(z) & z \in I_{+}
\end{array} \quad n \in \mathbb{N}\right.
$$

This leads to

$$
\left\langle e_{-r}, V e_{2 n}\right\rangle=-\frac{\mathrm{i}(-1)^{n}}{\pi} \cos \left(\frac{r \pi}{2}\right) \frac{1}{(2 n+r)(2 n+r+1)},
$$


so that

$$
\Sigma_{3}=\frac{64}{\pi^{4}} \sum_{n=1}^{\infty} \sum_{m=1}^{\infty}\left(\sum_{l=0}^{\infty} \sigma_{n, m, l}^{(3)}\right)^{2}
$$

where

$$
\sigma_{n, m, l}^{(3)}=\frac{(-1)^{l}}{(4 n-2 l+1)(4 n-2 l+3)(4 m+2 l+1)(4 m+2 l+3)} .
$$

Further,

$$
\begin{aligned}
& \Sigma_{4}=\left\|P_{2} V^{\prime *} S_{\mathrm{NS}} V P_{4}\right\|_{2}^{2}=\sum_{n=1}^{\infty} \sum_{m=1}^{\infty}\left|\sum_{r \in \mathbb{N}_{0}+\frac{1}{2}} \overline{\left\langle e_{-r}, V^{\prime} e_{2 n+1}\right\rangle}\left\langle e_{-r}, V e_{2 m}\right\rangle\right|^{2} \\
& =\frac{64}{\pi^{4}} \sum_{n=1}^{\infty} \sum_{m=1}^{\infty}\left(\sum_{l=0}^{\infty} \sigma_{n, m, l}^{(4)}\right)^{2}
\end{aligned}
$$

where

$$
\sigma_{n, m, l}^{(4)}=\frac{(-1)^{l}}{(4 n+2 l+3)(4 n+2 l+5)(4 m+2 l+1)(4 m+2 l+3)} .
$$

In the same way we compute

$$
\Sigma_{5}=\left\|P_{3} V^{*} S_{\mathrm{NS}} V P_{4}\right\|_{2}^{2}=\sum_{n=1}^{\infty} \sum_{m=1}^{\infty}\left|\sum_{r \in \mathbb{N}_{0}+\frac{1}{2}} \overline{\left\langle e_{-r}, V e_{-2 n}\right\rangle}\left\langle e_{-r}, V e_{2 m}\right\rangle\right|^{2} .
$$

The action of $V$ on basis vectors $e_{-2 n}$ is

$$
\left(V e_{-2 n}\right)(z)=\left\{\begin{array}{cc}
e_{-2 n}(z) & z \in I_{-} \\
-\mathrm{i} e_{-2 n-1}(z) & z \in I_{2} \\
-e_{-2 n}(z) & z \in I_{+}
\end{array} \quad n \in \mathbb{N} .\right.
$$

This leads us to

$$
\left\langle e_{-r}, V e_{-2 n}\right\rangle=\frac{\mathrm{i}(-1)^{n}}{\pi} \cos \left(\frac{r \pi}{2}\right) \frac{1}{(2 n-r)(2 n-r+1)},
$$

so that

$$
\Sigma_{5}=\frac{64}{\pi^{4}} \sum_{n=1}^{\infty} \sum_{m=1}^{\infty}\left(\sum_{l=0}^{\infty} \sigma_{n, m, l}^{(5)}\right)^{2}
$$

where

$$
\sigma_{n, m, l}^{(5)}=\frac{1}{(4 n-2 l-1)(4 n-2 l+1)(4 m+2 l+1)(4 m+2 l+3)} .
$$

Finally,

$$
\begin{aligned}
\Sigma_{6} & =\left\|P_{4} V^{*} S_{\mathrm{NS}} V P_{4}\right\|_{2}^{2}=\sum_{n=1}^{\infty} \sum_{m=1}^{\infty}\left|\sum_{r \in \mathbb{N}_{0}+\frac{1}{2}} \overline{\left\langle e_{-r}, V e_{2 n}\right\rangle}\left\langle e_{-r}, V e_{2 m}\right\rangle\right|^{2} \\
& =\frac{64}{\pi^{4}} \sum_{n=1}^{\infty} \sum_{m=1}^{\infty}\left(\sum_{l=0}^{\infty} \sigma_{n, m, l}^{(6)}\right)^{2}
\end{aligned}
$$


where

$$
\sigma_{n, m, l}^{(6)}=\frac{1}{(4 n+2 l+1)(4 n+2 l+3)(4 m+2 l+1)(4 m+2 l+3)} .
$$

Next, we turn to the discussion of the operator

$$
C=V S_{\mathrm{NS}} V^{*}-S_{\mathrm{R}}
$$

For showing that $\|C\|_{2}<\infty$ we prove that

$$
\left\|\left(\mathbf{1}-P_{0}\right) C\left(\mathbf{1}-P_{0}\right)\right\|_{2}=\left\|\sum_{i, j=1}^{4} P_{i} C P_{j}\right\|_{2} \leq \sum_{i, j=1}^{4}\left\|P_{i} C P_{j}\right\|_{2}<\infty .
$$

Because $C=C^{*}$ we have again only to treat those terms with $i \leq j$. Further, by

$$
\Gamma C \Gamma=V\left(\mathbf{1}-S_{\mathrm{NS}}\right) V^{*}-\left(\mathbf{1}-S_{\mathrm{R}}\right)=\left(V V^{*}-\mathbf{1}\right)-C
$$

and since $\mathbf{1}-V V^{*}$ is a rank one projection (i.e. $\left\|V V^{*}-\mathbf{1}\right\|_{2}=1$ ), we find

$$
\begin{aligned}
\left\|P_{1} C P_{1}\right\|_{2} & =\left\|\Gamma P_{1} C P_{1} \Gamma\right\|_{2}=\left\|P_{2} \Gamma C \Gamma P_{2}\right\|_{2}=\left\|P_{2}\left(V V^{*}-\mathbf{1}-C\right) P_{2}\right\|_{2} \\
& \leq\left\|P_{2} C P_{2}\right\|_{2}+1 .
\end{aligned}
$$

In the same way one obtains

$$
\left\|P_{3} C P_{3}\right\|_{2} \leq\left\|P_{4} C P_{4}\right\|_{2}+1, \quad\left\|P_{2} C P_{3}\right\|_{2} \leq\left\|P_{1} C P_{4}\right\|_{2}+1,
$$

and

$$
\left\|P_{1} C P_{3}\right\|_{2} \leq\left\|P_{2} C P_{4}\right\|_{2}+1 \text {. }
$$

Again, $S_{\mathrm{R}}$ is annihilated by $P_{2}$ or $P_{4}$ in these terms. Using once more that $V-V^{\prime}$ is Hilbert Schmidt class, we conclude that it is sufficient to prove the finiteness of the following six terms:

$$
\begin{array}{ccc}
\left\|P_{2} V S_{\mathrm{NS}} V^{*} P_{2}\right\|_{2}, & \left\|P_{1} V S_{\mathrm{NS}} V^{*} P_{2}\right\|_{2}, & \left\|P_{1} V S_{\mathrm{NS}} V^{\prime *} P_{4}\right\|_{2}, \\
\left\|P_{2} V S_{\mathrm{NS}} V^{\prime *} P_{4}\right\|_{2}, & \left\|P_{3} V^{\prime} S_{\mathrm{NS}} V^{\prime *} P_{4}\right\|_{2}, & \left\|P_{4} V^{\prime} S_{\mathrm{NS}} V^{\prime *} P_{4}\right\|_{2} .
\end{array}
$$

Now we have to work again,

$$
\Sigma_{7}=\left\|P_{2} V S_{\mathrm{NS}} V^{*} P_{2}\right\|_{2}^{2}=\sum_{n=1}^{\infty} \sum_{m=1}^{\infty}\left|\sum_{r \in \mathbb{N}_{0}+\frac{1}{2}} \overline{\left\langle e_{-r}, V^{*} e_{2 n+1}\right\rangle}\left\langle e_{-r}, V^{*} e_{2 m+1}\right\rangle\right|^{2} .
$$

The action of $V^{*}$ on basis vectors $e_{2 n+1}$ is

$$
\left(V^{*} e_{2 n+1}\right)(z)=\left\{\begin{array}{cc}
e_{2 n+1}(z) & z \in I_{-} \\
-\mathrm{i} e_{2 n}(z) & z \in I_{2} \\
-e_{2 n+1}(z) & z \in I_{+}
\end{array} \quad n \in \mathbb{N} .\right.
$$

This leads us to

$$
\left\langle e_{-r}, V^{*} e_{2 n+1}\right\rangle=-\frac{\mathrm{i}(-1)^{n}}{\pi} \sin \left(\frac{r \pi}{2}\right) \frac{1}{(2 n+r)(2 n+r+1)},
$$


so that

$$
\Sigma_{7}=\frac{64}{\pi^{4}} \sum_{n=1}^{\infty} \sum_{m=1}^{\infty}\left(\sum_{l=0}^{\infty} \sigma_{n, m, l}^{(7)}\right)^{2}
$$

where

$$
\sigma_{n, m, l}^{(7)}=\frac{1}{(4 n+2 l+1)(4 n+2 l+3)(4 m+2 l+1)(4 m+2 l+3)} .
$$

Further,

$$
\Sigma_{8}=\left\|P_{1} V S_{\mathrm{NS}} V^{*} P_{2}\right\|_{2}^{2}=\sum_{n=1}^{\infty} \sum_{m=1}^{\infty}\left|\sum_{r \in \mathbb{N}_{0}+\frac{1}{2}} \overline{\left\langle e_{-r}, V^{*} e_{-2 n-1}\right\rangle}\left\langle e_{-r}, V^{*} e_{2 m+1}\right\rangle\right|^{2} .
$$

The action of $V^{*}$ on basis vectors $e_{-2 n-1}$ is

$$
\left(V^{*} e_{-2 n-1}\right)(z)=\left\{\begin{array}{cl}
e_{-2 n-1}(z) & z \in I_{-} \\
\mathrm{i} e_{-2 n}(z) & z \in I_{2} \\
-e_{-2 n-1}(z) & z \in I_{+}
\end{array} \quad n \in \mathbb{N} .\right.
$$

This leads us to

$$
\left\langle e_{-r}, V^{*} e_{-2 n-1}\right\rangle=-\frac{\mathrm{i}(-1)^{n}}{\pi} \sin \left(\frac{r \pi}{2}\right) \frac{1}{(2 n-r)(2 n-r+1)},
$$

so that

$$
\Sigma_{8}=\frac{64}{\pi^{4}} \sum_{n=1}^{\infty} \sum_{m=1}^{\infty}\left(\sum_{l=0}^{\infty} \sigma_{n, m, l}^{(8)}\right)^{2}
$$

where

$$
\sigma_{n, m, l}^{(8)}=\frac{1}{(4 n-2 l-1)(4 n-2 l+1)(4 m+2 l+1)(4 m+2 l+3)} .
$$

Further,

$$
\Sigma_{9}=\left\|P_{1} V S_{\mathrm{NS}} V^{\prime *} P_{4}\right\|_{2}^{2}=\sum_{n=1}^{\infty} \sum_{m=1}^{\infty}\left|\sum_{r \in \mathbb{N}_{0}+\frac{1}{2}} \overline{\left\langle e_{-r}, V^{*} e_{-2 n-1}\right\rangle}\left\langle e_{-r}, V^{\prime *} e_{2 m}\right\rangle\right|^{2} .
$$

The action of $V^{\prime *}$ on basis vectors $e_{2 n}$ is

$$
\left(V^{\prime *} e_{2 n}\right)(z)=\left\{\begin{array}{cl}
e_{2 n}(z) & z \in I_{-} \\
-\mathrm{i} e_{2 n-1}(z) & z \in I_{2} \\
-e_{2 n}(z) & z \in I_{+}
\end{array} \quad n \in \mathbb{N} .\right.
$$

This leads us to

$$
\left\langle e_{-r}, V^{\prime *} e_{2 n}\right\rangle=\frac{\mathrm{i}(-1)^{n}}{\pi} \cos \left(\frac{r \pi}{2}\right) \frac{1}{(2 n+r)(2 n+r-1)},
$$

so that

$$
\Sigma_{9}=\frac{64}{\pi^{4}} \sum_{n=1}^{\infty} \sum_{m=1}^{\infty}\left(\sum_{l=0}^{\infty} \sigma_{n, m, l}^{(9)}\right)^{2}
$$


where

$$
\sigma_{n, m, l}^{(9)}=\frac{(-1)^{l}}{(4 n-2 l-1)(4 n-2 l+1)(4 m+2 l-1)(4 m+2 l+1)} .
$$

Further,

$$
\begin{aligned}
\Sigma_{10} & =\left\|P_{2} V S_{\mathrm{NS}} V^{\prime *} P_{4}\right\|_{2}^{2}=\sum_{n=1}^{\infty} \sum_{m=1}^{\infty}\left|\sum_{r \in \mathbb{N}_{0}+\frac{1}{2}} \overline{\left\langle e_{-r}, V^{*} e_{2 n+1}\right\rangle}\left\langle e_{-r}, V^{\prime *} e_{2 m}\right\rangle\right|^{2} \\
& =\frac{64}{\pi^{4}} \sum_{n=1}^{\infty} \sum_{m=1}^{\infty}\left(\sum_{l=0}^{\infty} \sigma_{n, m, l}^{(10)}\right)^{2}
\end{aligned}
$$

where

$$
\sigma_{n, m, l}^{(10)}=\frac{(-1)^{l}}{(4 n+2 l+1)(4 n+2 l+3)(4 m+2 l-1)(4 m+2 l+1)} .
$$

Further,

$$
\Sigma_{11}=\left\|P_{3} V^{\prime} S_{\mathrm{NS}} V^{\prime *} P_{4}\right\|_{2}^{2}=\sum_{n=1}^{\infty} \sum_{m=1}^{\infty}\left|\sum_{r \in \mathbb{N}_{0}+\frac{1}{2}} \overline{\left\langle e_{-r}, V^{\prime *} e_{-2 n}\right\rangle}\left\langle e_{-r}, V e_{2 m}\right\rangle\right|^{2} .
$$

The action of $V^{\prime *}$ on basis vectors $e_{-2 n}$ is

$$
\left(V^{\prime *} e_{-2 n}\right)(z)=\left\{\begin{array}{cl}
e_{-2 n}(z) & z \in I_{-} \\
\mathrm{i} e_{-2 n+1}(z) & z \in I_{2} \\
-e_{-2 n}(z) & z \in I_{+}
\end{array} \quad n \in \mathbb{N} .\right.
$$

This leads us to

$$
\left\langle e_{-r}, V^{\prime *} e_{-2 n}\right\rangle=-\frac{\mathrm{i}(-1)^{n}}{\pi} \cos \left(\frac{r \pi}{2}\right) \frac{1}{(2 n-r)(2 n-r-1)},
$$

so that

$$
\Sigma_{11}=\frac{64}{\pi^{4}} \sum_{n=1}^{\infty} \sum_{m=1}^{\infty}\left(\sum_{l=0}^{\infty} \sigma_{n, m, l}^{(11)}\right)^{2}
$$

where

$$
\sigma_{n, m, l}^{(11)}=\frac{1}{(4 n-2 l-3)(4 n-2 l-1)(4 m+2 l-1)(4 m+2 l+1)} .
$$

Finally,

$$
\begin{aligned}
\Sigma_{12} & =\left\|P_{4} V^{\prime} S_{\mathrm{NS}} V^{\prime *} P_{4}\right\|_{2}^{2}=\sum_{n=1}^{\infty} \sum_{m=1}^{\infty}\left|\sum_{r \in \mathbb{N}_{0}+\frac{1}{2}} \overline{\left\langle e_{-r}, V^{\prime *} e_{2 n}\right\rangle}\left\langle e_{-r}, V^{\prime *} e_{2 m}\right\rangle\right|^{2} \\
& =\frac{64}{\pi^{4}} \sum_{n=1}^{\infty} \sum_{m=1}^{\infty}\left(\sum_{l=0}^{\infty} \sigma_{n, m, l}^{(12)}\right)^{2}
\end{aligned}
$$


where

$$
\sigma_{n, m, l}^{(12)}=\frac{1}{(4 n+2 l-1)(4 n+2 l+1)(4 m+2 l-1)(4 m+2 l+1)} .
$$

We have the following estimate of absolute values of the $\sigma_{n, m, l}^{(j)}$ for $j=1,4,6,7$, 10, 12:

$$
\sigma_{n, m, l}^{(1)} \geq\left|\sigma_{n, m, l+2}^{(j)}\right|, \quad j=4,6,7,10,12, \quad n, m \in \mathbb{N}, \quad l \in \mathbb{N}_{0} .
$$

If we omit in our summations $l=0$ and $l=1$ terms, this corresponds to the replacement of $S_{\mathrm{NS}}$ by

$$
S_{\mathrm{NS}}^{\prime}=S_{\mathrm{NS}}-\left|e_{-\frac{1}{2}}\right\rangle\left\langle e_{-\frac{1}{2}}|-| e_{-\frac{3}{2}}\right\rangle\left\langle e_{-\frac{3}{2}}\right| .
$$

Since the difference $S_{\mathrm{NS}}-S_{\mathrm{NS}}^{\prime}$ is obviously Hilbert Schmidt class, this has no influence of the property of $\Sigma_{j}$ to be finite or infinite. Hence the estimate (80) tells us that for the proof of $\Sigma_{j}<\infty, j=1,4,6,7,10,12$, it is sufficient to prove it for $j=1$. We compute

$$
\begin{aligned}
\Sigma_{1} & =\frac{64}{\pi^{4}} \sum_{n, m=1}^{\infty}\left(\sum_{l=0}^{\infty} \frac{1}{(4 n+2 l+3)(4 n+2 l+5)(4 m+2 l+3)(4 m+2 l+5)}\right)^{2} \\
& <\frac{64}{\pi^{4}} \sum_{n=1}^{\infty} \sum_{m=1}^{\infty} \frac{1}{(2 m+1)^{4}}\left(\sum_{l=0}^{\infty} \frac{1}{(4 n+2 l+3)^{2}}\right)^{2} \\
& =\frac{2}{3} \sum_{n=1}^{\infty}\left(\sum_{l=0}^{\infty} \frac{1}{(4 n+2 l+3)^{2}}\right)^{2} \\
& \leq \frac{2}{3} \sum_{n=1}^{\infty}\left(\int_{0}^{\infty} \frac{d l}{(4 n+2 l+1)^{2}}\right)^{2} \\
& =\frac{2}{3} \sum_{n=1}^{\infty}\left(\frac{1}{2(4 n+1)}\right)^{2} \\
& <\frac{1}{96} \sum_{n=1}^{\infty} \frac{1}{n^{2}} \\
& =\frac{\pi^{2}}{576}
\end{aligned}
$$

$\Sigma_{1}$ is finite. On the other hand we find for $n, m \in \mathbb{N}, l \in \mathbb{N}_{0}$

$$
\begin{aligned}
\left|\sigma_{n, m, l}^{(11)}\right|>\left|\sigma_{n, m, l+2}^{(2)}\right|, & \left|\sigma_{n, m, l}^{(11)}\right|>\left|\sigma_{n, m, l+2}^{(3)}\right|, & \left|\sigma_{n, m, l}^{(11)}\right|>\left|\sigma_{n, m, l+1}^{(5)}\right|, \\
\left|\sigma_{n, m, l}^{(11)}\right|>\left|\sigma_{n, m, l+1}^{(8)}\right|, & \left|\sigma_{n, m, l}^{(11)}\right|>\left|\sigma_{n, m, l+1}^{(9)}\right| . &
\end{aligned}
$$

By the same argument, for the proof of $\Sigma_{j}<\infty, j=2,3,5,8,9,11$, it is sufficient to prove that

$$
\tilde{\Sigma}_{11}=\frac{64}{\pi^{4}} \sum_{n=1}^{\infty} \sum_{m=1}^{\infty}\left(\sum_{l=0}^{\infty}\left|\sigma_{n, m, l}^{(11)}\right|\right)^{2}<\infty .
$$


For this purpose, we decompose the sum over the index $l$ into three parts,

$$
\begin{aligned}
\sum_{l=0}^{\infty}\left|\sigma_{n, m, l}^{(11)}\right| & =\sum_{l=0}^{2 n-2}\left|\sigma_{n, m, l}^{(11)}\right|+\left|\sigma_{n, m, 2 n-1}^{(11)}\right|+\sum_{l=2 n}^{\infty}\left|\sigma_{n, m, l}^{(11)}\right| \\
& =\sum_{l=0}^{2 n-2} \sigma_{n, m, l}^{(11)}-\sigma_{n, m, 2 n-1}^{(11)}+\sum_{l=2 n}^{\infty} \sigma_{n, m, l}^{(11)} .
\end{aligned}
$$

We begin with estimating the first part. By reversing the order of summation we obtain

$$
\begin{aligned}
& \sum_{l=0}^{2 n-2} \sigma_{n, m, l}^{(11)}= \\
& =\sum_{l=0}^{2 n-2} \frac{1}{(2 l+1)(2 l+3)(4 m+4 n-2 l-3)(4 m+4 n-2 l-5)} \\
& <\sum_{l=0}^{2 n+2 m-3} \frac{1}{(2 l+1)^{2}(4 n+4 m-2 l-5)^{2}} \\
& =\sum_{l=0}^{n+m-2} \frac{1}{(2 l+1)^{2}(4 n+4 m-2 l-5)^{2}} \\
& +\sum_{l=n+m-1}^{2 n+2 m-3} \frac{1}{(2 l+1)^{2}(4 n+4 m-2 l-5)^{2}} \\
& =2 \sum_{l=0}^{n+m-2} \frac{1}{(2 l+1)^{2}(4 n+4 m-2 l-5)^{2}} \\
& \leq \frac{2}{(4 n+4 m-5)^{2}}+2 \int_{0}^{n+m-2} \frac{d l}{(2 l+1)^{2}(4 n+4 m-2 l-5)^{2}} \\
& =\frac{2}{(4 n+4 m-5)^{2}}+\int_{1}^{2 n+2 m-3} \frac{d x}{x^{2}(4 n+4 m-4-x)^{2}} \\
& =\frac{2}{(4 n+4 m-5)^{2}}+\frac{1}{(4 n+4 m-4)^{2}(2 n+2 m-1)} \\
& -\frac{1}{(4 n+4 m-4)^{2}(4 n+4 m-5)} \\
& -\frac{1}{(4 n+4 m-4)(2 n+2 m-3)(2 n+2 m-1)} \\
& +\frac{1}{(4 n+4 m-4)(4 n+4 m-5)}-\frac{2}{(4 n+4 m-4)^{3}} \ln \left(\frac{2 n+2 m-1}{2 n+2 m-3}\right) \\
& +\frac{2}{(4 n+4 m-4)^{3}} \ln (4 n+4 m-5) \\
& <\frac{6}{(2 n+2 m-3)^{2}} \text {. }
\end{aligned}
$$

The last estimate is very rough but correct. In our computation we have used the fact that in an area of strict decrease a summation can be estimated by an 
integral plus the first summand. Next we consider the only negative term,

$$
-\sigma_{n, m, 2 n-1}^{(11)}=\frac{1}{(4 n+4 m-3)(4 n+4 m-1)}<\frac{1}{(2 n+2 m-3)^{2}},
$$

and finally the remaining summation,

$$
\begin{aligned}
\sum_{l=2 n}^{\infty} \sigma_{n, m, l}^{(11)} & =\sum_{l=0}^{\infty} \frac{1}{(2 l+1)(2 l+3)(4 n+4 m+2 l-1)(4 n+4 m+2 l+1)} \\
& <\frac{1}{(4 n+4 m-1)^{2}} \sum_{l=0}^{\infty} \frac{1}{(2 l+1)(2 l+3)} \\
& =\frac{1}{(4 n+4 m-1)^{2}} \\
& <\frac{1}{(2 n+2 m-3)^{2}} .
\end{aligned}
$$

We now can conclude that

$$
\tilde{\Sigma}_{11}<\frac{64}{\pi^{4}} \sum_{n=1}^{\infty} \sum_{m=1}^{\infty}\left(\frac{8}{(2 n+2 m-3)^{2}}\right)^{2}=\frac{4096}{\pi^{4}} \sum_{k=0}^{\infty} \frac{k+1}{(2 k+1)^{4}}<\infty
$$

the proof of Lemma 3.10 is complete, q.e.d.

acknowledgement I am grateful to Prof. K. Fredenhagen for discussions. He supported this investigation with many ideas. Thanks are also due to C. Binnenhei for a careful reading of the manuscript.

\section{References}

[1] Araki, H.: On Quasifree States of CAR and Bogoliubov Automorphisms. Publ. RIMS Kyoto Univ. Vol. 6 (1970/71)

[2] Araki, H.: Bogoliubov Automorphisms and Fock Representations of the Canonical Anticommutation Relations. In: Operator Algebras and Mathematical Physics, Am. Math. Soc. Vol. 62 (1987)

[3] Araki, H., Evans, D.E.: On a $C^{*}$-Algebra Approach to Phase Transitions in the Two-Dimensional Ising Model. Commun. Math. Phys. 91 (1983)

[4] Binnenhei, C.: Implementation of Endomorphisms of the CAR algebra. SFB 288 preprint No. 142. Submitted to Rev. Math. Phys. (1994)

[5] Böckenhauer, J.: Lokale Normalität und lokalisierte Endomorphismen des chiralen Ising-Modells. Diplomarbeit, Hamburg (1994)

[6] Böckenhauer, J.: Decomposition of Representations of CAR Induced by Bogoliubov Endomorphisms. DESY 94-173 (1994)

[7] Brunetti, R., Guido, D., Longo, R.: Modular Structure and Duality in Conformal Quantum Field Theory. Commun. Math. Phys. 156 (1993)

[8] Buchholz, D., Mack, G., Todorov, I.: Localized Automorphisms of the U(1)-Current Algebra on the Circle: An Instructive Example. In: [19] 
[9] Buchholz, D., Schulz-Mirbach, H.: Haag Duality in Conformal Quantum Field Theory. Rev. Math. Phys. 2 (1990)

[10] Doplicher, S., Haag, R., Roberts, J.E.: Fields, Observables and Gauge Transformations I \& II. Commun. Math. Phys. 13 (1969) and 15 (1969)

[11] Doplicher, S., Haag, R., Roberts, J.E.: Local Observables and Particle Statistics I \& II. Commun. Math. Phys. 23 (1971) and 35 (1974)

[12] Douglas, R.G.: Banach Algebra Techniques in Operator Theory. Academic Press, New York, London (1972)

[13] Fredenhagen, K., Rehren, K.-H., Schroer, B.: Superselection Sectors with Braid Group Statistics and Exchange Algebras I. Commun. Math. Phys. 125 (1989)

[14] Fredenhagen, K., Rehren, K.-H., Schroer, B.: Superselection Sectors with Braid Group Statistics and Exchange Algebras II. SFB 288 preprint No. 10 (1992)

[15] Fredenhagen, K.: Quantum Field Theory on Nontrivial Spacetimes. To appear in: Proceedings of the Beersheva Conference 1993, R. Sen (Hrsg.)

[16] Fuchs, J., Ganchev, A., Vecsernyés, P.: Level 1 WZW Superselection Sectors. Commun. Math. Phys. 146 (1992)

[17] Haag, R.: Local Quantum Physics. Springer-Verlag, Berlin, Heidelberg, New York (1992)

[18] Haag, R., Kastler, D.: An Algebraic Approach to Quantum Field Theory. J. Math. Phys. 5 (1964)

[19] Kastler, D. (ed.): The Algebraic Theory of Superselection Sectors. World Scientific, Singapur (1990)

[20] Lang, S.: $\mathrm{SL}_{2}(\mathbb{R})$. Springer-Verlag, Berlin, Heidelberg, New York (1985)

[21] Loke, T.: Operator Algebras and Conformal Field Theory of the Discrete Series Representations of Diff $\left(S^{1}\right)$. Dissertation, Cambridge (1994)

[22] Lüscher, M., Mack, G.: The Energy Momentum Tensor of Critical Quantum Field Theories in 1+1 Dimensions. Unpublished manuscript (1976)

[23] Mack, G., Schomerus, V.: Conformal Field Algebras with Quantum Symmetry from the Theory of Superselection Sectors. Commun. Math. Phys. 134 (1990)

[24] Mack, G., Schomerus, V.: Endomorphisms and Quantum Symmetry of the Conformal Ising Model. In: 19.

[25] Powers, R.T., Størmer, E.: Free States of the Canonical Anticommutation Relations. Commun. Math. Phys. 16 (1970)

[26] Reed, M., Simon, B.: Methods of Modern Mathematical Physics. Vol. 1: Functional Analysis. Academic Press, New York, London (1972) 
[27] Rideau, G.: On Some Representations of the Anticommutation Relations. Commun. Math. Phys. 9 (1968)

[28] Schroer, B.: A Trip to Scalingland. In: Ferreira, E. (ed.): V. Brazilian Symposium on Theoretical Physics, Vol. I (1974)

[29] Szlachányi, K.: Chiral Decomposition as a Source of Quantum Symmetry in the Ising Model. KFKI-1993-16/A preprint (1993)

[30] Szlachányi, K.: The Universal Algebra of Local Even CAR and Majorana Algebras on the Circle. Unpublished manuscript (1993)

[31] Wassermann, A.: Operator Algebras and Conformal Field Theory. To appear in: Proceedings of the International Congress of Mathematicians, Zürich 1994, Birkhäuser Verlag 